\title{
DEVELOPMENT OF A SILICON NANOWIRE MASK USING SCANNING PROBE MICROSCOPY
}

\author{
A Thesis \\ presented to \\ the Faculty of the California Polytechnic State University, \\ San Luis Obispo
}

\author{
In Partial Fulfillment \\ of the Requirements for the Degree \\ Master of Science in Materials Engineering
}

by

Ross Gregoriev

August, 2014 
(C) 2014

Ross Gregoriev

ALL RIGHTS RESERVED 


\section{COMMITTEE MEMBERSHIP}

TITLE:

Development of a Silicon Nanowire Mask using Scanning Probe Microscopy

AUTHOR:

Ross Gregoriev

DATE SUBMITTED: August 2014

COMMITTEE CHAIR Dr. Richard Savage

Professor of Materials Engineering

California Polytechnic State University,

San Luis Obispo

COMMITTEE MEMBER: Dr. Derek Gragson

Professor of Chemistry

California Polytechnic State University,

San Luis Obispo

COMMITTEE MEMBER: Dr. Gregory Scott

Assistant Professor of Chemistry

California Polytechnic State University,

San Luis Obispo 


\begin{abstract}
Development of a Silicon Nanowire Mask using Scanning Probe Microscopy Ross Gregoriev
\end{abstract}

Scanning probe microscopy techniques were used to investigate the desorption of hydrogen passivated silicon to form $\mathrm{SiO}_{2}$ etch masks. The application of the etch masks were planned on being used to manufacture silicon nanowires. Low concentration hydrofluoric acid was used to passivate the surface. The surface was selectively depassivated by SPM techniques. Scanning tunneling microscopy (STM) and atomic force microscopy (AFM) were used to create the masks. The STM system used was found to desorb hydrogen from the surface faster than the STM could image and was considered incapable in the configuration investigated. This led to the use of atomic force microscopy (AFM). Using a conductive tip in contact mode, lithography on the passivated surface was performed. The topography of the lithography was compared to similar works and found to be similar in size. The width was found to be $80 \mathrm{~nm}$ and the thickness $1 \mathrm{~nm}$. The depassivated layers were confirmed to be oxide through electronic force microscopy (EFM). Finally, voltages were swept with the tip in contact with the surface to find the bandgap of the oxide. It was found that the voltage sweeps were severely modifying the tip along with producing inconsistent desorption thicknesses ranging from 0.2 to $12 \mathrm{~nm}$. Despite the results from the voltage sweeps, the lithography procedure performed using the AFM was found to be successful. 


\section{ACKNOWLEDGMENTS}

I would like to thank my advisor, Dr. Savage, for helping me with resources and learning tools crucial to the success of the project. I would also like to thank Dr. Scott for his help and assistance with the AFM and STM. 


\section{TABLE OF CONTENTS}

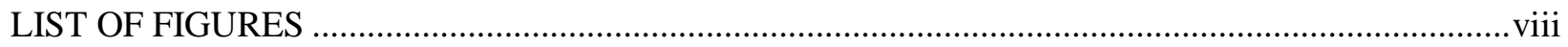

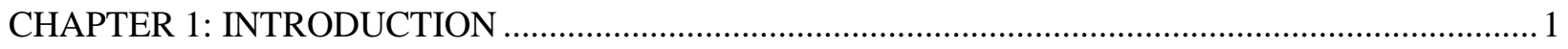

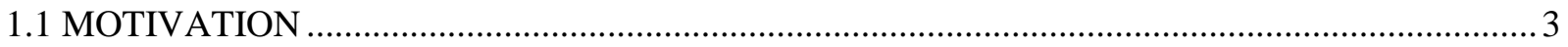

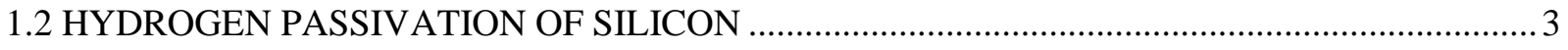

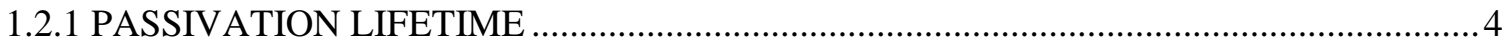

1.2.2 DESORPTION AND OXIDATION OF PASSIVATED SILICON SURFACES ................4

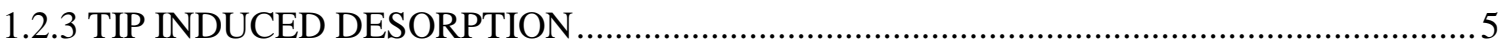

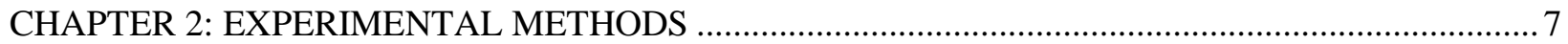

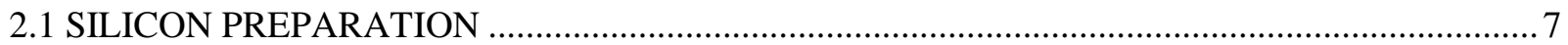

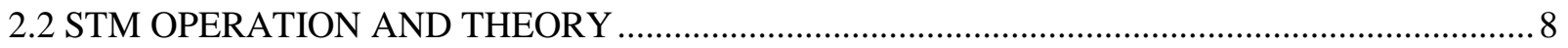

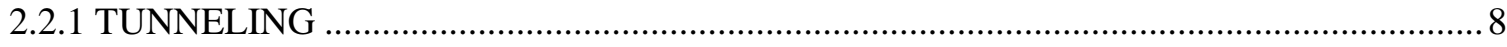

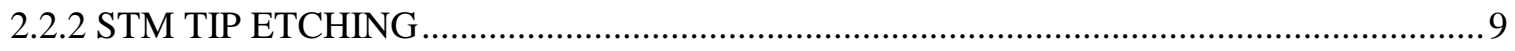

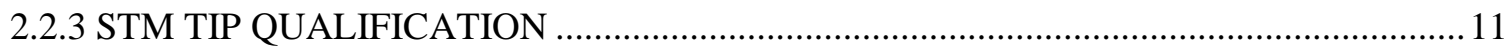

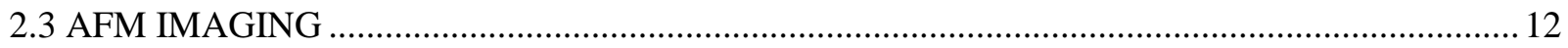

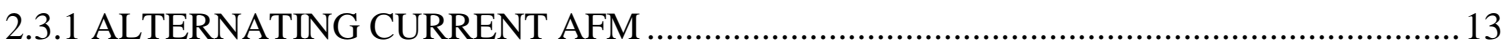

2.3.2 PHASE LOCKED LOOPS IN TAPPING MODE AFM IMAGING APPLICATIONS...... 14

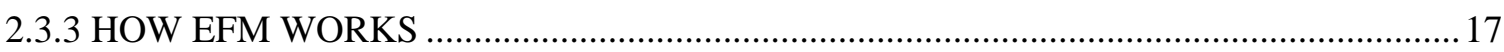

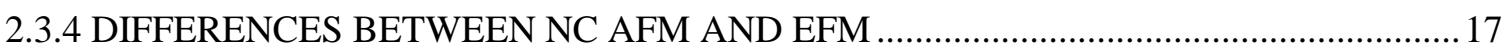

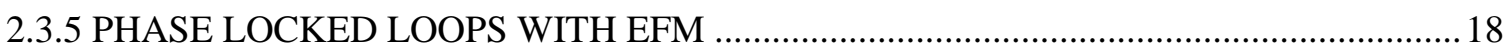

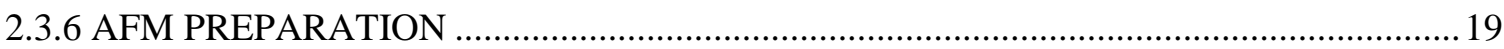

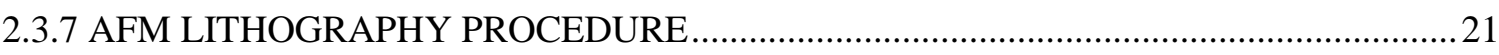

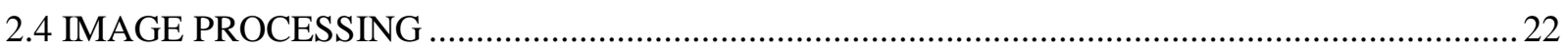

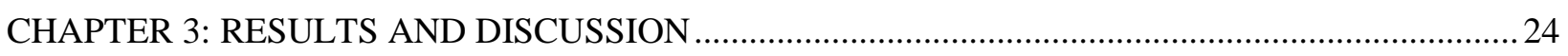

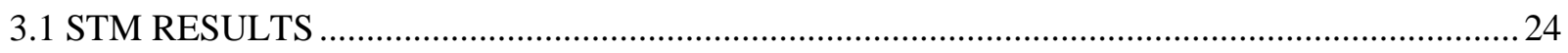

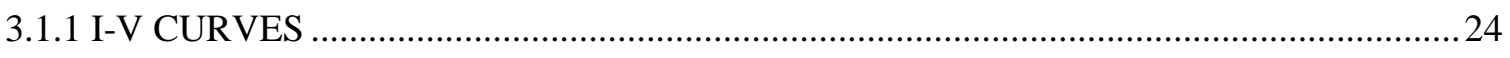

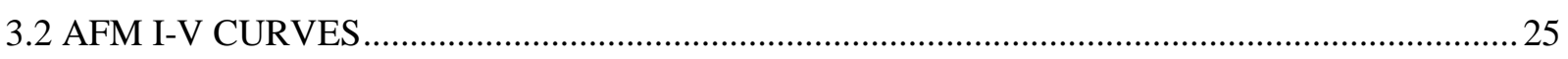

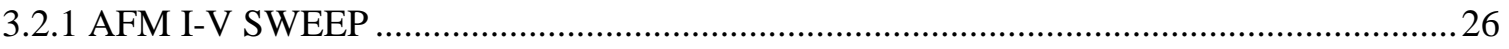

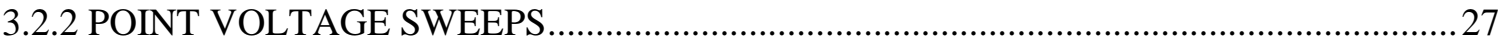

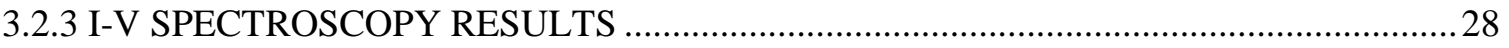

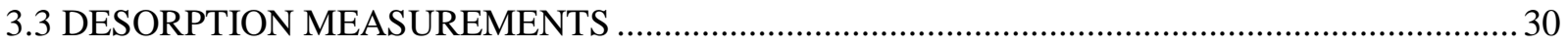

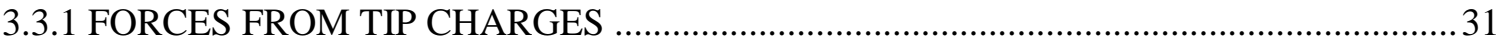

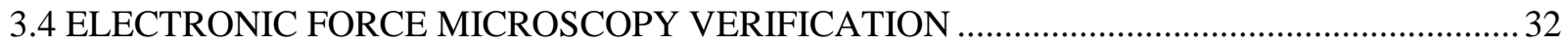




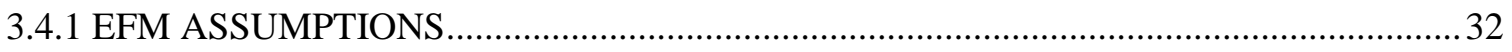

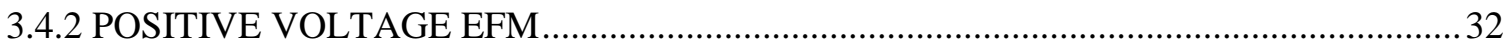

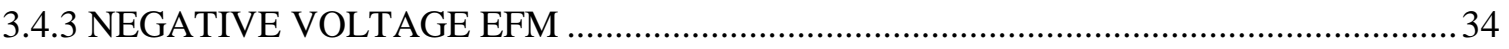

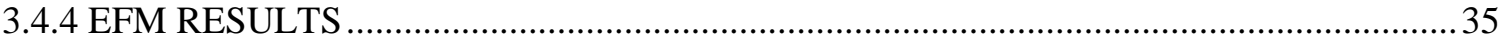

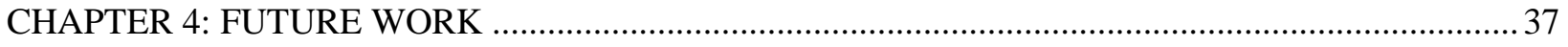

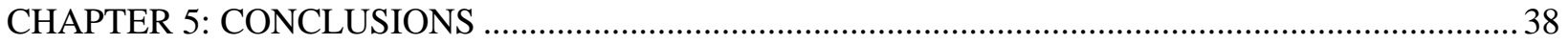

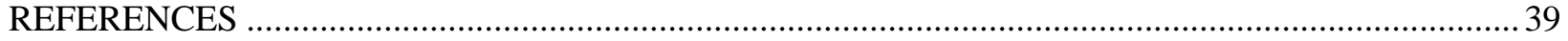




\section{LIST OF FIGURES}

Figure 1: Layout of a typical nanowire transistor. ............................................................................ 1

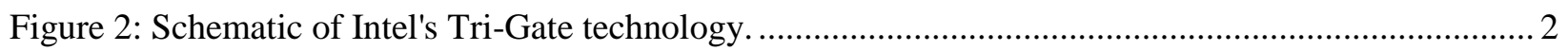

Figure 3: Schematic of cutoff circuit (left) along with the setup of the etcher (right). ............................ 10

Figure 4: Atomically resolved HOPG with verification of atom spacing typical of a sharp tip................ 11

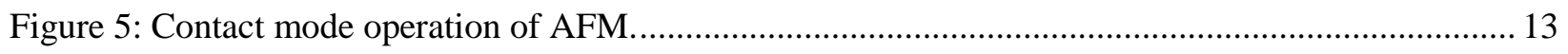

Figure 6: Potential Energy curve representing the forces involved when the tip approaches the sample

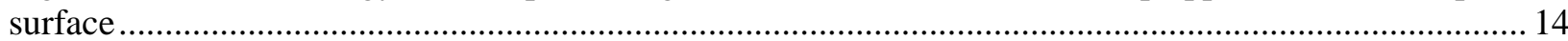

Figure 7: The interactions between the tip and the sample cause a phase and amplitude shift as seen

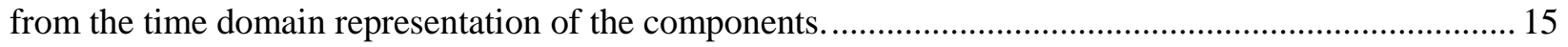

Figure 8: Sine wave properties arising from the I and Q components ................................................. 16

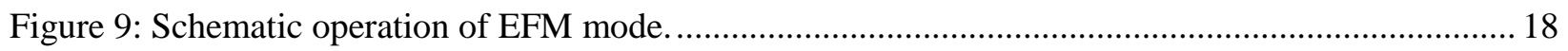

Figure 10: Visual representation of how frequency change from the tip's interactions with the sample

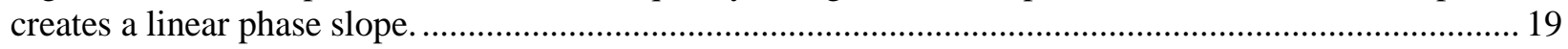

Figure 11: A cantilever inserted into the "ORCA" cantilever holder. ...................................................... 20

Figure 12: Diced silicon sample with sample holder on the scanning stage .......................................... 21

Figure 13: Superimposed I-V curves to compare the gradual increase of electrical resistance. Time 1 represents initial exposure time. Times 2,3,4 were performed at 8,13, and 17 minutes after the first

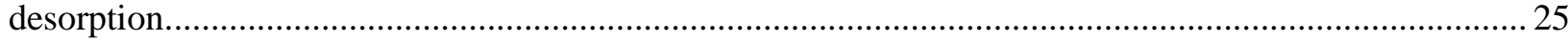

Figure 14: Current vs. Voltage sweep with the voltage applied to the surface, modulated from -3V to $3 \mathrm{~V}$

Figure 15: Current vs. Voltage sweeps performed on multiple passivated areas. The sweeps show the

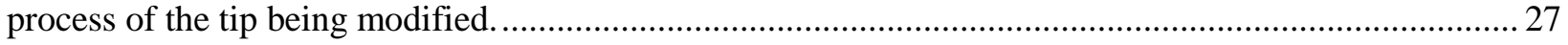

Figure 16: Scans after performing coarse voltage point spectroscopy. The topography after applying negative voltage a) showed no presence of oxide. b) An EFM scan shows there was residual charge. c)

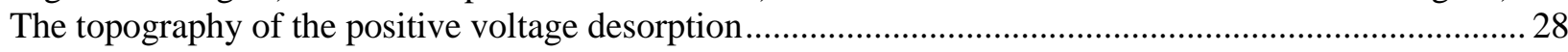

Figure 17: Tapping mode scan after performing coarse voltage point spectroscopy...............................29

Figure 18: Extracted Height profiles from both the rough and fine voltage sweeps................................ 30

Figure 19: Cal Poly logo drawn though desorption. a) The topography of the scan is shown along with the location for the line profile extraction. b) The corresponding step height measurement. 31

Figure 20: Charge interaction between the tip and sample when performing EFM with a positive surface bias..

Figure 21: Charge interactions between the tip and sample when performing EFM with a negative surface bias.

Figure 22: EFM images on the edge of a desorbed square. The left side of the step is the semiconductor while the right is a part of the desorbed square. The EFM images correspond to a surface voltage of a) $0 \mathrm{~V}, \mathrm{~b})-2 \mathrm{~V}$, and c) $1 \mathrm{~V}$. 


\section{CHAPTER 1: INTRODUCTION}

Nanotechnology is a burgeoning technology that allows for electronics to not only get smaller and faster, but also cheaper and more efficient. An example of how nanoscale devices are increasing computational power while reducing power consumption is through nanowire transistors. Nanowire transistors use a semiconducting nanowire across two conductors to form a transistor (Figure 1). When an electric field is applied to the gate, the semiconductor reacts by allowing current to flow from the drain to the source

\section{Nanowire Transistor}

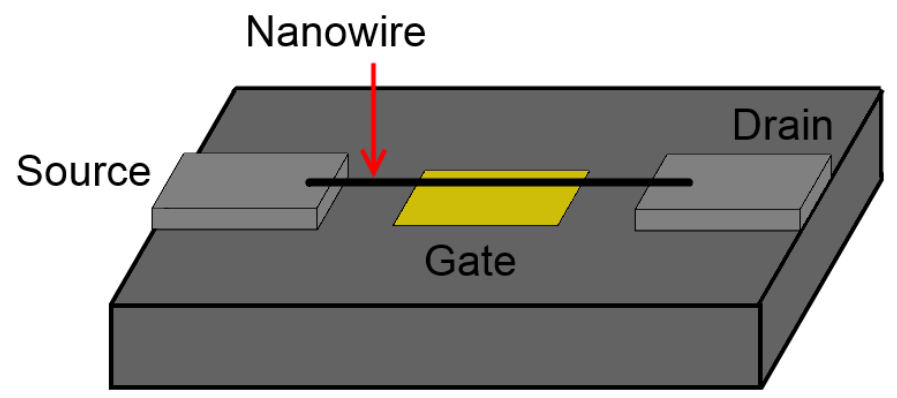

Figure 1: Layout of a typical nanowire transistor.

A current application where a nanowire-like operation is being used to increase computational power is Intel's Tri-Gate technology ${ }^{1}$. The Tri-Gate technology is a deviation from the standard planar transistor technology that creates flat transistors on top of silicon (Figure 2). The Tri-Gate structure utilizes a semiconducting fin with a gate oxide over the semiconductor. With this configuration, $22 \mathrm{~nm}$ node size transistors have been implemented in the Ivy Bridge processor architecture. 


\section{Intel's Tri-Gate Transistor}

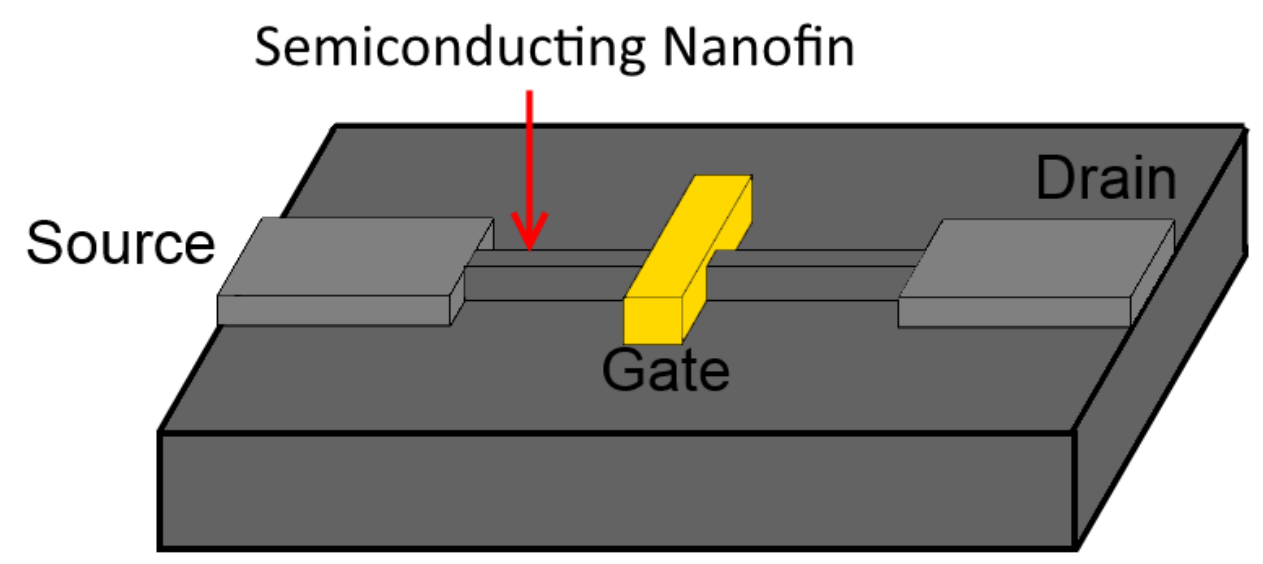

Figure 2: Schematic of Intel's Tri-Gate technology.

One method of producing nanoscale masks on substrates is using dip pen nanolithography ${ }^{2,3}$. The lithography is performed by treating an AFM cantilever with a polymer. One can think of the treatment as the action of dipping a quill pen in ink to store ink in the pen. There are many polymers that can be used to treat the cantilevered tip, but the line width corresponds to the size of the polymers. The treated cantilever is then pressed into the sample to allow for the polymer to transfer through capillary action. The resulting layer of polymer can then undergo chemical processes using the polymer as a mask that will act as a chemical barrier to protect the material under the mask, also known as an etch resist.

An example of a dip pen nanolithography when applied to nanowire transistors is the use of the procedure to create the drain and source of a transistor ${ }^{3}$. This method patterns a polymer to act as a gold etch resist. The polymer was drawn on to allow for semiconducting carbon nanotubes to be bound to two isolated gold pads. Once the device was etched, the transistor was complete. The advantage of this sort of 
manufacturing is that many polymers can be used, and the application of the polymer is versatile. The disadvantage of this method is that the locations of the transistors are not chosen, which is impractical for creating high density, compact transistors.

\subsection{MOTIVATION}

The purpose of this paper is to investigate the creation of nanoscale masks using scanning probe microscopy (SPM) to produce nanowire etch masks. This involves understanding the factors contributing to the physics and kinetics of growing an oxide from a hydrogen passivated silicon surface.

Manufacturing of silicon nanowire masks were investigated using a scanning tunneling microscope (STM) and an atomic force microscope (AFM). The data acquired from creating the nanowire masks were compared against values found in literature to determine the success of the lithography procedure.

The specific process that was investigated was top-down manufacturing of silicon nanowires. The top-down method utilizes an oxide mask on the surface. Utilizing the oxide's chemical resistance compared to the substrate, channels with widths of $50 \mathrm{~nm}$ can be produced by etching away the surrounding silicon ${ }^{4}$. The specific process that is investigated in this paper is the creation of the oxide masks by means of replacing a hydrogen passivated layer with silicon oxide to be used as an etch resist to produce silicon nanowires ${ }^{5}$. The passivation layer is a metastable layer comprised of silicon hydride. The hydrogen is selectively removed through electrical interactions with a scanning probe microscope. The dangling bonds that are created oxidize in the presence of water and oxygen. The oxide can then be grown through the diffusion of oxygen through the surface oxide.

\subsection{HYDROGEN PASSIVATION OF SILICON}

The process of passivating silicon occurs when silicon is exposed to hydrofluoric acid. The native oxide present on the surface of the silicon undergoes the following reaction:

$$
\mathrm{SiO}_{2}+6 \mathrm{HF}->\mathrm{H}_{2}+\mathrm{SiF}_{6}+2 \mathrm{H}_{2} \mathrm{O}
$$


This reaction strips the surface of silicon oxide. The hydrogen present in the HF is able to bond with the dangling bonds of the silicon atoms. The result is a metastable phase consisting of silicon hydride. The metastable phase will slowly desorb if left in ambient conditions.

\subsubsection{PASSIVATION LIFETIME}

After passivating the silicon surface, the lifespan of the hydrogen monolayer was found to be a critical aspect for performing lithography using the hydrogen desorption method. The lifespan needed to be long enough that lithography, verification, and etching procedures could be performed before the layer entirely desorbs. The desorption timeframe was found be heavily dependent on the presence of humidity

${ }^{4,7-9}$. Laboratory conditions in which lithography was performed were at $40 \%-45 \%$. At $40 \%$ humidity, the passivated layer has been shown to last around 10 hours before a significant coverage of oxide was present ${ }^{7}$. When performing lithography, the layer stops producing clearly defined features after 3 to 5 hours of lithography and analysis. The lifetime of the passivation layer was found to be suitable to complete procedures upon, but some aspects could decrease the working time. The desiccant chamber could have leaked atmospheric air into the chamber which provided extra moisture to desorb the passivation ${ }^{10}$. The presence of ultraviolet light from the sun contributes to desorption of the monolayer by stimulating the silicon atom through absorption of a high enough energy photon.

\subsubsection{DESORPTION AND OXIDATION OF PASSIVATED SILICON SURFACES}

The desorption process is a multi-step process that replaces the hydrogen bonds with oxygen ${ }^{7}$. The passivated surface is comprised of silicon hydride due to the dangling bonds present on the oxide stripped surface of the (100) silicon. Once the surface is exposed to an oxidative atmosphere, intermediate compounds of $\mathrm{SiH}_{2} \mathrm{O}_{2}$ and $\mathrm{SiHO}_{3}$ begin to form as the surface atoms react with oxygen. Finally the hydrogen desorbs and ambient oxygen binds to the surface to form an oxide layer. The $\mathrm{SiO}_{2}$ then acts as a permeable surface for oxygen to pass through and allow for additional growth of the oxide layer if sufficient energy is present. 
In atmospheric conditions, a meniscus of water tends to form on the surface of the sample being imaged. The humidity in the air contributes to the moisture layer that forms on top of the silicon in atmospheric conditions. This moisture's presence is proven later in the paper. The aspect ratio of the grown oxide has been shown to be heavily based on the humidity of the air ${ }^{7,8}$.

The structure of the surface in each phase can be determined by macroscopic property changes of the surface. When the surface is mostly comprised of hydrogen terminated bonds, the electronic forces between the hydrogen on the silicon and the oxygen atom in water are repulsive. This repulsion force makes the surface hydrophobic. As the hydrogen is replaced with oxygen atoms, the oxygen atom on the surface can combine with water to produce a hydroxide $(\mathrm{OH})$ ion attached to the silicon surface ${ }^{7}$. The hydroxide on the surface will then make the surface hydrophilic. From these properties, it is easy to determine the presence of a passivated surface by determining if the surface is hydrophobic or hydrophilic. This verification step has one weakness, the water applied can also assist desorption and prematurely desorb the hydrogen.

\subsubsection{TIP INDUCED DESORPTION}

Lithography was performed through the removal of the hydrogen passivation layer from the surface. This was achieved by exposing the passivation layer to a potential. The added energy of the potential allows for desorption to occur much faster than just desorption due to atmospheric conditions. There are two methods that can cause desorption: vibrational stimulation of the $\mathrm{SiH}$ bond and electronic stimulation of the $\mathrm{SiH}$ bond $^{11,12}$. The vibrational stimulation is generated for $\mathrm{n}$ type silicon when the surface is biased negative with respect to the tip. The electronic stimulation is generated for $\mathrm{n}$ type silicon when the surface has a positive bias with respect to the tip. The vibrational method utilizes the holes generated from flowing current from the silicon to the tip. These holes generate phonons that can carry enough energy to thermally detach the $\mathrm{SiH}$ bond. This method requires more energy to desorb and has a wide desorption area. 
The electron stimulation utilizes the interaction of the electrons with the silicon to sever the hydrogen bond. The energy can arrive in two methods: tunneling or field emission. The difference between these two methods is the oxide thickness and width. The tunneling method can be used to desorb individual atoms, but the tunneling probability goes down significantly as the oxide gets thicker. The incapability of electrons to penetrate the barrier limits the growth of the oxide. This method is the desorption mechanism for positive voltages up to $4 \mathrm{~V}^{12}$. At this point, the oxide stops growing. The field emission can be used to penetrate the surface oxide in order to grow a thicker oxide, but doing so increases trace width of the desorption. The trace width is widened through the increased surface area on the tip emitting electrons to the sample. This mode of operation occurs when there a potential of greater than $4 \mathrm{~V}^{12}$. The field emission method was found to have the best characteristics for creating masks, and was chosen to be used. 


\section{CHAPTER 2: EXPERIMENTAL METHODS}

\subsection{SILICON PREPARATION}

The lithography procedure begins with passivation of silicon. The silicon used was an ntype (100) wafer. The reasoning for using this type of silicon is that it is available and cost effective. The goal of the sample preparation is to remove the surface oxide and coat the surface with silicon hydride. The devised method uses a combination of ultrasonic, plasma treatment and pre hydrofluoric acid (HF) dips. The hydrofluoric acid used was a buffered oxide etchant (BOE) that has a buffer of ammonium fluoride added to maintain a constant $\mathrm{HF}$ concentration ${ }^{6}$. A class 1000 clean room was used to prepare the samples to help reduce contamination while preparing the samples. Any introduced contamination (e.g. dropping the silicon sample) at any points of the procedure resulted in an immediate rejection of the sample.

The deionized water used for the cleaning procedures was verified to have over $15 \mathrm{M} \Omega \cdot \mathrm{cm}$ in resistivity. The resistivity of the water is proportional to the metal ion contaminates in the water. Low resistance water can desorb the passivated surface. Then, the beaker used for the final deionized water quench was ultrasonically cleaned using the $15 \mathrm{M} \Omega \cdot \mathrm{cm}$ water for at least two minutes. The beaker was subjected to three ultrasonic cleaning cycles to ensure complete removal of any residual chemicals and material in the beaker so that the measured resistance of the deionized water used was the same as the resistance of the water used in the quench. The high-resistance deionized water allows for the silicon hydride layer to remain intact while the residual BOE is removed.

The cleaning steps of the silicon sample intended to remove surface contaminates. The silicon samples were initially cleaned by ultrasonic cleaning in isopropanol to clean the surface of organic contaminates. The samples were put in a beaker filled with isopropanol and put in an ultrasonic bath for at least 5 minutes. After, the samples were rinsed with $15 \mathrm{M} \Omega \cdot \mathrm{cm}$ water and dried using low purity nitrogen gas. The samples were then dipped in a BOE bath for 30 seconds to remove the oxide layer on the surface. The sample was then dipped the sample into the $15 \mathrm{M} \Omega \cdot \mathrm{cm}$ quench bath and dried with nitrogen. 
A 30 second plasma treatment was then performed using an argon plasma gun, removing the surface layer through etching. The result of this process is a clean surface ready to be passivated.

The passivation process utilizes the same BOE and quench baths used in cleaning. The samples were taken from the surface stripped state and dipped in BOE for 30 seconds. The samples were then dipped in the same deionized water used to clean the sample and dried using nitrogen. The samples were then immediately stored in a vacuum of 25 torr within a desiccant chamber. The desiccant chamber was used to increase the lifespan of the surface so that the samples can be transported to the AFM located outside of the clean room. When the surface is exposed to atmospheric conditions, the passivation layer will gradually desorb. Given enough time, the native oxide layer regrows and desorbs all the hydrogen from the surface ${ }^{7}$.

\subsection{STM OPERATION AND THEORY}

\subsubsection{TUNNELING}

The scanning tunneling microscope uses phenomena known as tunneling to transmit current between two materials without actual contact ${ }^{13}$. The separation between the tip and the sample is known as the tunneling barrier. In order to cross the barrier classically, the energy of the electron needed to be greater than the energy associated with the barrier (barrier height). From Schrodinger's equation from quantum mechanics, it can be proved that there is a probability for which an electron with insufficient energy will cross the barrier. The advantage of using the tunneling effect is that the tunneling probability is exponentially decreased when increasing the barrier width. Therefore, a sharp enough tip can establish a tunneling current that interacts solely with individual atoms. When the tunneling current established is maintained on the STM and the tip is rastered, atomic scale measurements can be performed.

The STM system used was a Nanosurf Easyscan STM system on top of a TS150 active vibration isolation system. The tips used to image the silicon were electrochemically etched tungsten. 
The tunneling current setpoints ranged from 50pA to $2 \mathrm{nA}$. The voltages used to image silicon ranged from -2 to 2 volts with the bias originating from the tip.

\subsubsection{STM TIP ETCHING}

The STM system utilizes a tungsten tip that is sharpened to atomic sharpness using an electrochemical etch setup. The setup is seen in Figure 3 and utilizes 2 molar $\mathrm{KOH}$ as an electrolyte to reduce tungsten to $\mathrm{WO}_{3}$ and dissolve it into the solution. The drop method in which the tip is formed is a mechanical tip formation. Tungsten wire is submerged in the electrolyte with a $10 \mathrm{~mm}$ diameter gold cathode ring concentric to the wire. A voltage of 3.6 volts is applied to the ring to start the etching process. The etching process showed to have an accelerated etch rate at the bottom of the meniscus. The accelerated etch rate allows for the tungsten to neck below the meniscus and cause a stress concentration. Once the cross sectional area of the neck becomes too small to support the rest of the submerged tungsten, the tungsten breaks from tension in the neck and elongates the tungsten to make an atomically sharp tip. At the same moment the tip is created, etching can begin on the freshly cleaved tip. To prevent etching that would dull the tip, current was cut rapidly. At the moment of breakage, the current through the electrolytic cell drops rapidly. A cutoff circuit is developed to cut the current within $500 \mathrm{~ns}$ of drop off detection. Using an oscilloscope, the performance of the etch cutoff was verified to be less around 200ns, providing sufficient current cutoff speed. 
The schematic of the tip etching electronics is shown in Figure 3. The applied voltage goes to the cathode of the tip etcher and the anode goes into the $100 \mathrm{ohm}$ resistor. This resistor is used to convert current through the system to voltage using Ohms Law where voltage is equal to the current multiplied by the resistance $(\mathrm{V}=\mathrm{IR})$. The output of the resistor goes to the source pin of the MOSFET. The MOSFET controls the flow of current though the etching system. When the gate is driven low by the microcontroller, the current is cut from the circuit and the tip etcher is disconnected from ground so that no more current can flow. When the gate pin is high, current can flow freely. The drop off detection was determined by a Schmitt trigger. A Schmitt trigger is a comparator that will compare two voltages and indicate if an input voltage is higher or lower than a reference voltage. The special part of the Schmitt trigger is that there is an included hysteresis to reduce unnecessary switching if the input voltage comes close to the reference voltage. The input voltage is determined through the voltage drop across the resistor that represents the current flow. The reference voltage is set via the microcontroller and was programmed to be one quarter of the power supply. This voltage represented a low current, so that the tip etcher would not prematurely stop etching the tungsten if the tip cross sectional area became small enough. The cutoff voltage corresponded to about $4 \mathrm{~mA}$ depending on the excitation voltage. A typical etch would start with current flowing at about $15 \mathrm{~mA}$. At about $7 \mathrm{~mA}$, the neck will break, exposing the fresh tip. At drop off, the current is cut in about 200ns.
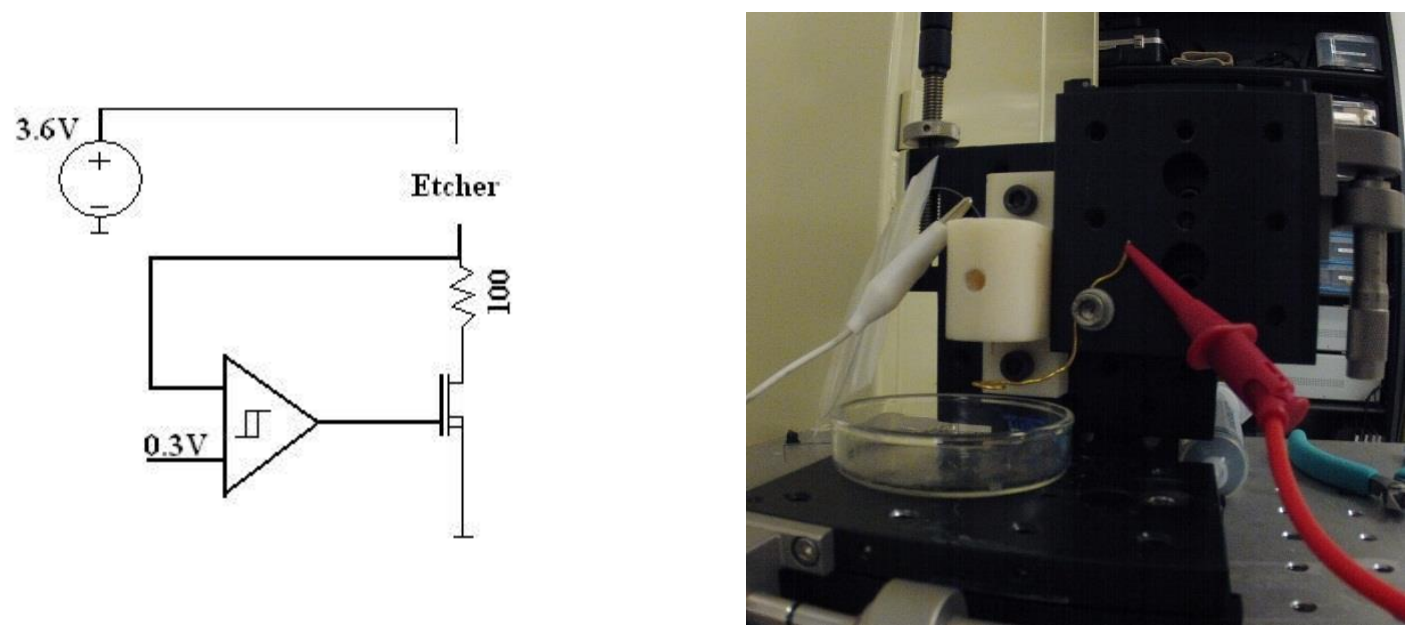

Figure 3: Schematic of cutoff circuit (left) along with the setup of the etcher (right). 


\subsubsection{STM TIP QUALIFICATION}

To verify that the tip was suitable for STM operation, the tip was tested with a sample of highly organized pyrolitic graphite (HOPG). A poorly etched tip has multiple tunneling points that will cycle randomly, making it impossible to acquire an image. A tip can be considered adequately sharp if a single point on the tip is used to tunnel resulting in the capability to accurately resolve the atomic structure of the sample. The tip used for spectroscopy was found to have a sharp enough point where each carbon atom of the HOPG able to be imaged (Figure 4). Along with verifying the sharpness of the tip, a test for double tips was performed by measuring the distance between the carbon atoms to verify that the distance between atoms is $260 \mathrm{pm}$. The tips used were found to satisfy the requirements for an accurate tunneling capable tip.

Once the tip was verified as a functional tip, the silicon sample was mounted to a magnetic

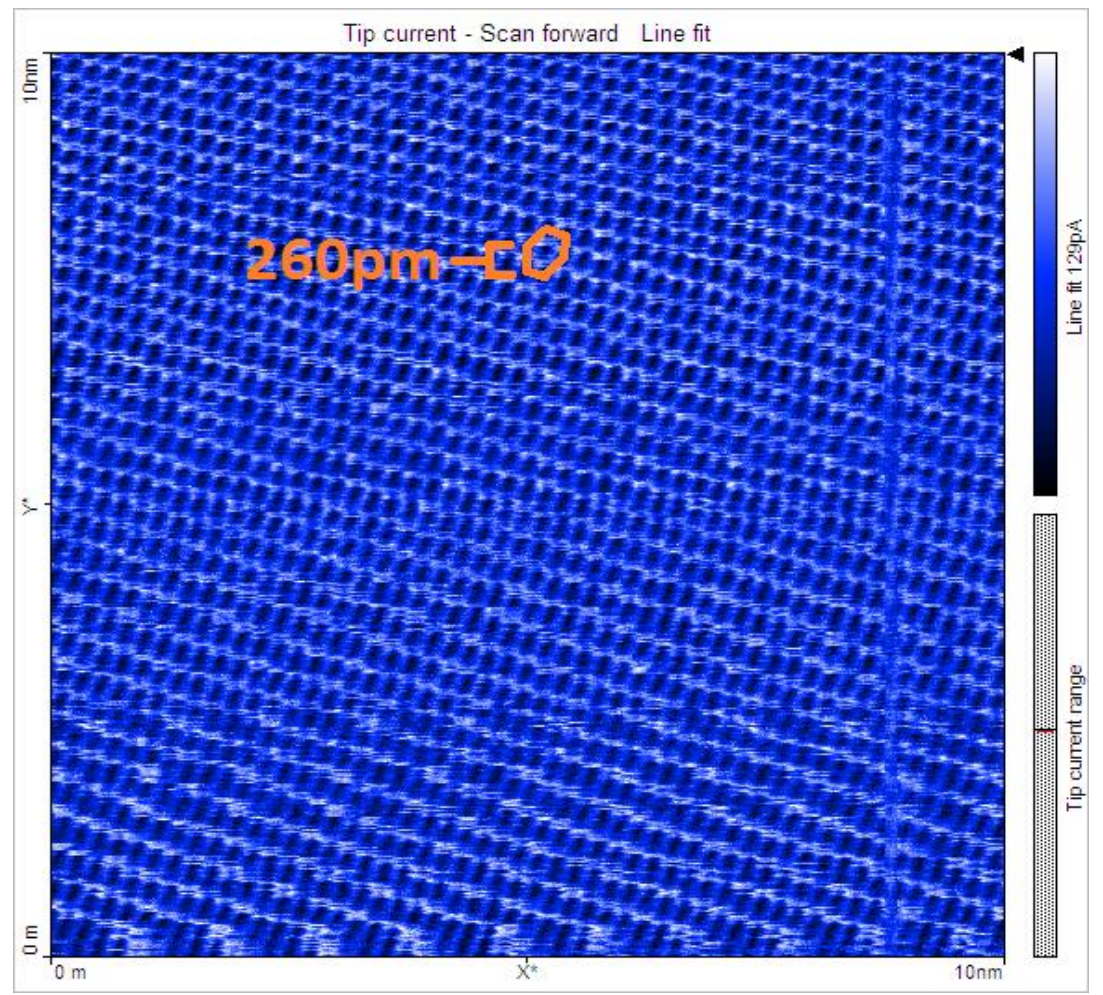

Figure 4: Atomically resolved HOPG with verification of atom spacing typical of a sharp tip. 
sample holder through conductive carbon tape. In addition to the carbon tape, colloidal silver would be used to ensure electrical connectivity. Once the sample was brought near the tip of the STM, an initial image was attempted to be acquired with settings of $-1 \mathrm{~V}$ at $-2 \mathrm{nA}$ current set point. If the result of this scan was noise, spectroscopy was then performed in the same area to investigate the reason for the noise.

\subsection{AFM IMAGING}

The operation of the AFM is shown in Figure 5. The tip is rastered across the sample and the cantilever is deflected by forces generated by the sample. The deflection of the cantilever causes a change in the laser pathway that is picked up by a photosensitive diode (PSD). As the tip rasters across the surface, a $3 \mathrm{~d}$ mapping of the sample is created.

The AFM utilizes a feedback loop to control the height of the cantilever to assist with the height measurement. There is a piezo actuator that raises and lowers the tip to prevent the tip from being rammed into the sample and breaking the tip. A piezo actuator is crystalline material that is able to convert voltage into physical movements. The advantage of using piezos is that the movement resolution can be less than the diameter of an atom. The height adjustment piezo is controlled with a proportional integral derivative controller (PID). While the operation of the PID theory is beyond the scope of the paper, the integral gain is used to "focus" the image by adjusting how fast the z-axis will react to height change. 


\section{AFM Standard Operation}

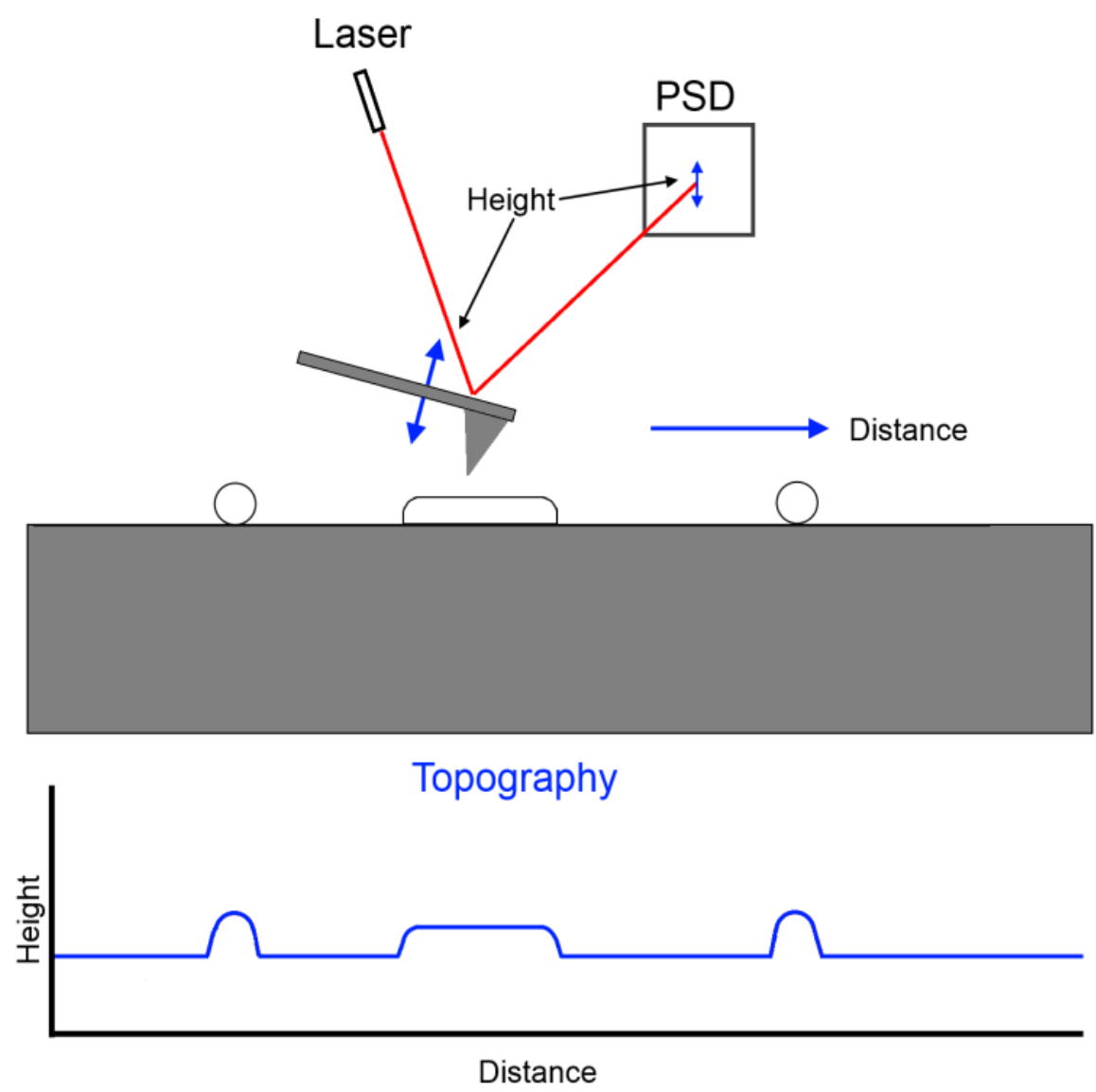

Figure 5: Contact mode operation of AFM.

\subsubsection{ALTERNATING CURRENT AFM}

There are multiple modes of operation to the Atomic Force Microscope. The mode used to image the samples after desorption is by using the alternating current (AC) mode. The alternating current refers to the cantilever being driven at a frequency using an alternating voltage source. The advantage of using a frequency-based tracking method is that higher resolution can be obtained by minimizing the interactions between the tip and the sample.

AC mode AFM utilizes interatomic forces as a feedback mechanism to control the height of the tip. The oscillating tip responds to Van Der Waals forces as the atoms approach each other. The interactions can be described using a potential energy curve (Figure 6). If the interactions used are 
attractive in nature, the AFM is operating in noncontact mode. This mode approaches but does not touch the surface for imaging. The attractive forces generated in this mode reduce the operating frequency of the tip. If the interactions are repulsive, the AFM is in tapping mode. This mode uses the repulsive force generated from the tip atoms physically interacting with the sample. The repulsive force increases the operating frequency of the cantilever. The change in frequency is measured by the phase locked loop (PLL) in the AFM.

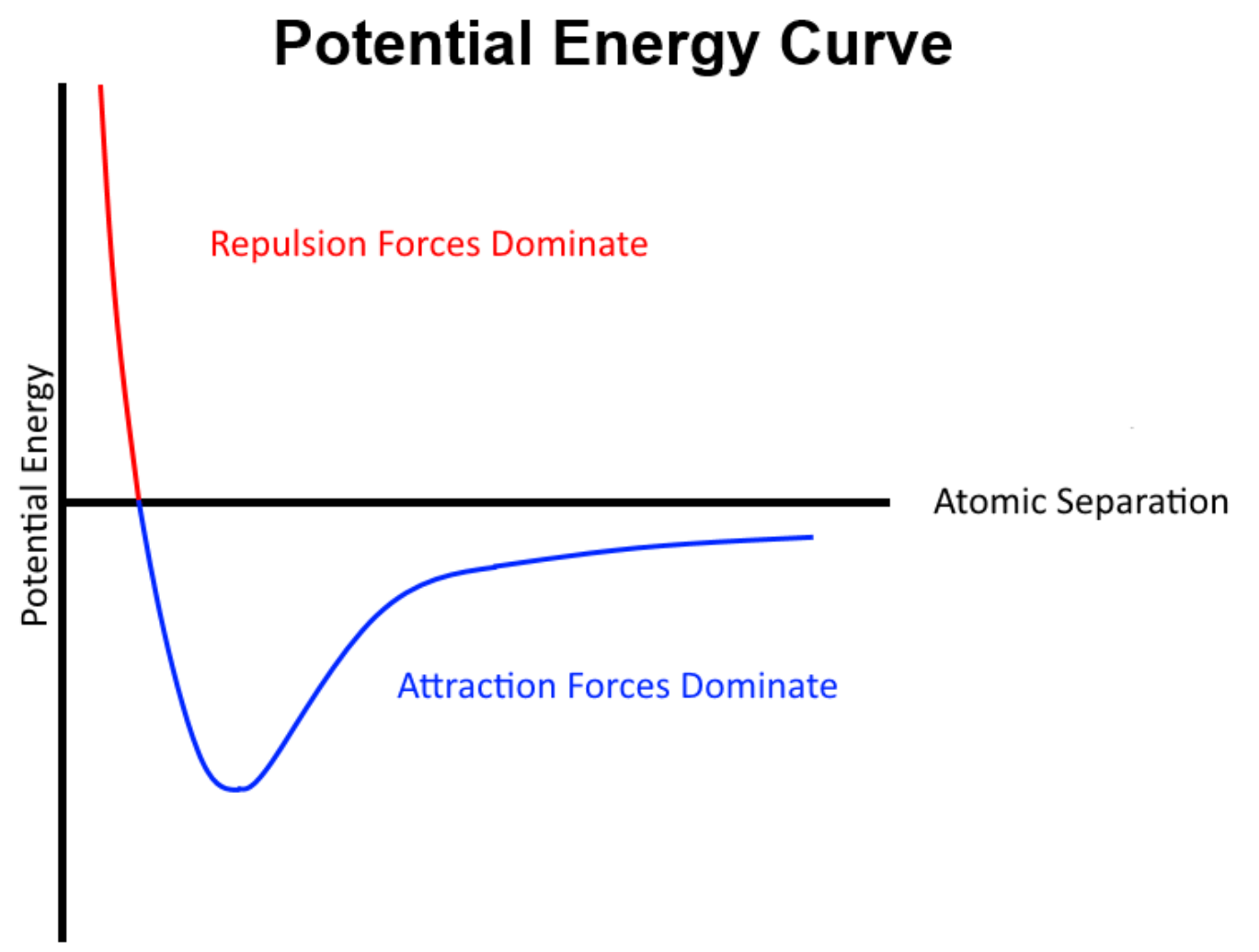

Figure 6: Potential Energy curve representing the forces involved when the tip approaches the sample surface

\subsubsection{PHASE LOCKED LOOPS IN TAPPING MODE AFM IMAGING APPLICATIONS}

The key component involved with measuring surface charge is the AFM's phase lock loop (PLL) termed as a "Digital Lock-In" system that measures differences in amplitude and phase between a reference oscillation and a signal oscillation. The equation representing amplitude of the oscillation in the time domain shown as 


$$
y=A \cdot \cos (2 \pi f t+\varphi)
$$

where A represents the amplitude of the oscillation, $f$ represents the frequency of oscillation and $\varphi$ represents the phase offset of the angle. On the AFM, the two time domain oscillations compared are: oscillating the tip using an AC voltage applied to the piezo oscillator, and the PSD that reads out the laser interaction with the tip. An example input signal with the phase and amplitude components labeled is shown in Figure 7. These shifts in phase and amplitude represent the interaction of the tip with the sample.

The PLL operates by extracting the in-phase and quadrature components of the acquired signal from the oscillating tip. An analog phase detector is used to process a cosine and sine wave that represents the real component (in-phase) of the signal's oscillation and the imaginary component (quadrature) of the signal's oscillation respectively. The cosine wave is used to drive the shake piezo at the tip's natural frequency. Both the cosine and sine waves are multiplied by the incoming signal from the PSD. The resulting signals are passed through a low pass filter to eliminate the excitation frequency and

\section{Phase and Amplitude}

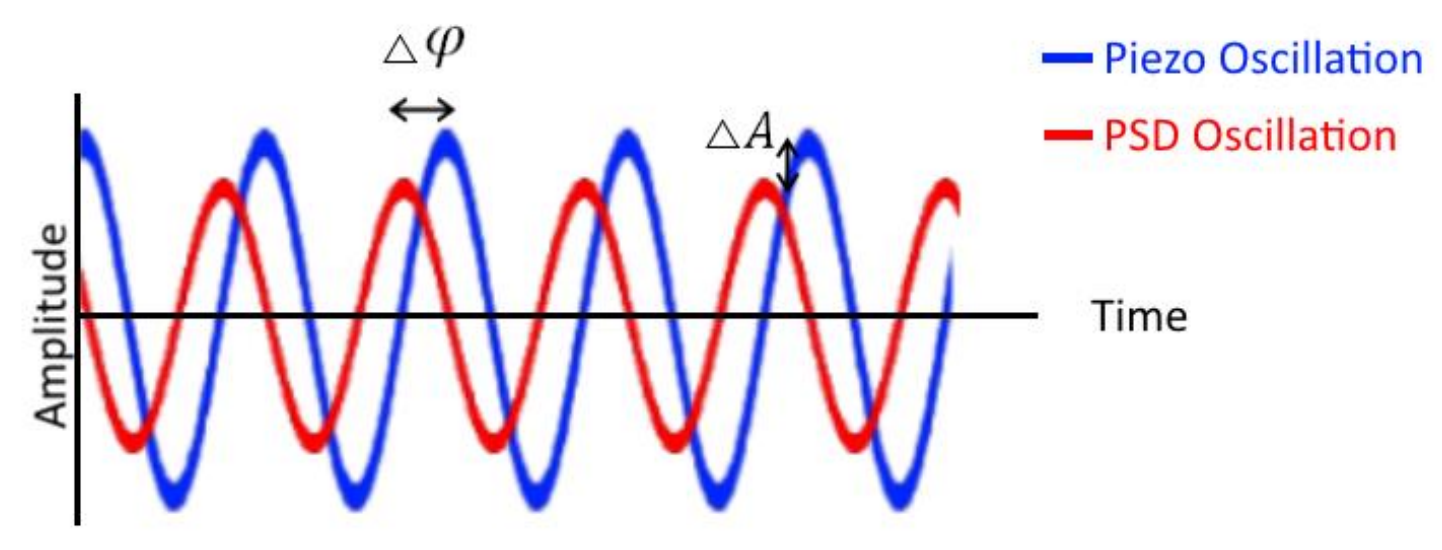

Figure 7: The interactions between the tip and the sample cause a phase and amplitude shift as seen from the time domain representation of the components. 
eliminate the $2 \pi f t$ term. The resulting two signals represent the phase and amplitude of the oscillating tip.

From the in-phase (I) and quadrature (Q) components of the oscillating sample, the amplitude and phase of the oscillation can be found. The representation of the signal's oscillation properties is shown in Figure 8 . The amplitude can be extracted using the magnitude of the in-phase and quadrature components. The phase angle can be extracted by measuring the angle between the in-phase component and the quadrature components.

\section{I-Q Cordinate System}

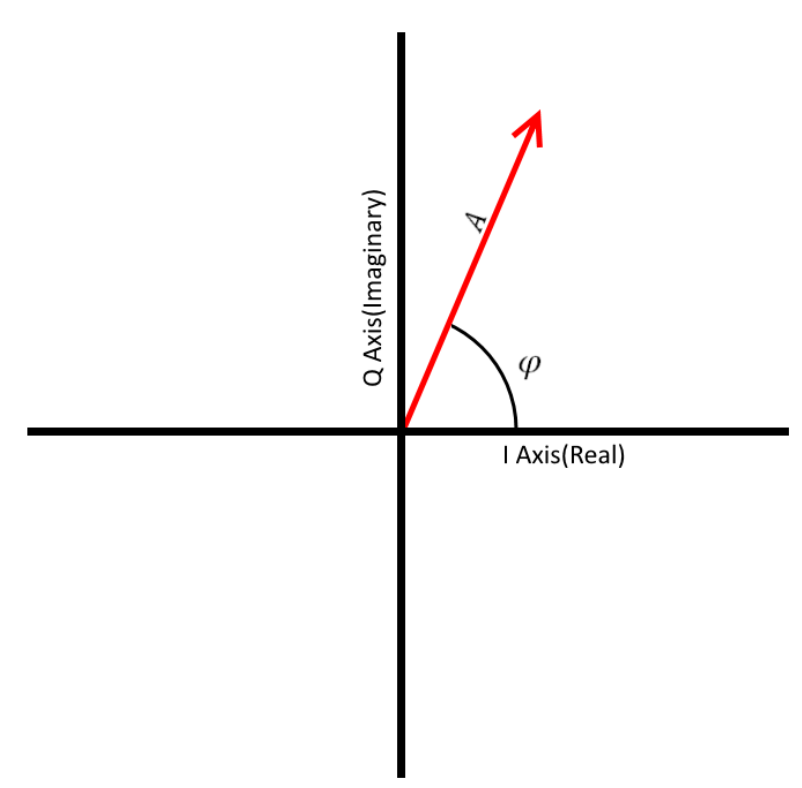

Figure 8: Sine wave properties arising from the I and Q components

The AFM uses the Phase Locked Loop as the feedback mechanism when operating in AC mode.

The phase locked loop locks on to a near-resonant frequency of the cantilever determined by the operator. The tip is then advanced towards the surface until there is a change in the resonant frequency. The change in frequency will cause a change in the amplitude of the oscillation. The feedback will adjust the tip height to counteract the change in the measured amplitude. The speed of the phase locked loop is seen on amplitude scans. When there are increased interactions as a result of the tip interacting with a protrusion, the error of the operating frequency will spike the amplitude. The feedback tries to maintain constant 
amplitude, so the tip will be raised or lowered to adjust for the amplitude change. This feedback mode allows for the tip to resolve topography with minimal interactions with the surface.

\subsubsection{HOW EFM WORKS}

Electron Force Microscopy is a modified operation of standard tapping mode AFM. The mode adds a second scan to the standard tapping mode scan. The extra scan follows the original scan contour but will offset the tip a nominal distance above the surface as determined by the operator. This type of scan is referred to as a Nap scan. As the tip follows the offset topography, a constant voltage is applied to the tip. When properly tuned, this allows for the electrical interactions to be the dominant interactions over the Van der Waals forces. As a result, the change in frequencies from the interacting electric fields creates a phase map that represents the electrical behavior of the surface at each point in the scan. From these behaviors, the relative conductivity of targets on the surface can be obtained. Finding the relative conductivities is important in determining the presence of an oxide on the surface by distinguishing the lower electrical conductivity of the insulator from the relatively conductive n-type silicon.

\subsubsection{DIFFERENCES BETWEEN NC AFM AND EFM}

The Nap scan is exactly like an offset tapping mode AFM scan with a few differences. A schematic representation of the EFM scanning mode is shown in Figure 9. The lock-in amplifier is freed up to be used to acquire electronic forces when the Nap scan follows the previous height profile directly. This also means the feedback in the Z-axis is disabled. The contour is assumed to be correct so the phase measurement can be used to determine the result of electrical forces while excluding the Van der Waals forces. The Nap's phase is taken from the lock-in amplifier and represents the electronic forces involved between the tip and the sample. These forces alter the operational frequency between the tip and sample depending on the charges between the surface and the tip. The PLL locks onto the phase to measure the frequency shifts. 


\section{EFM on an AFM}

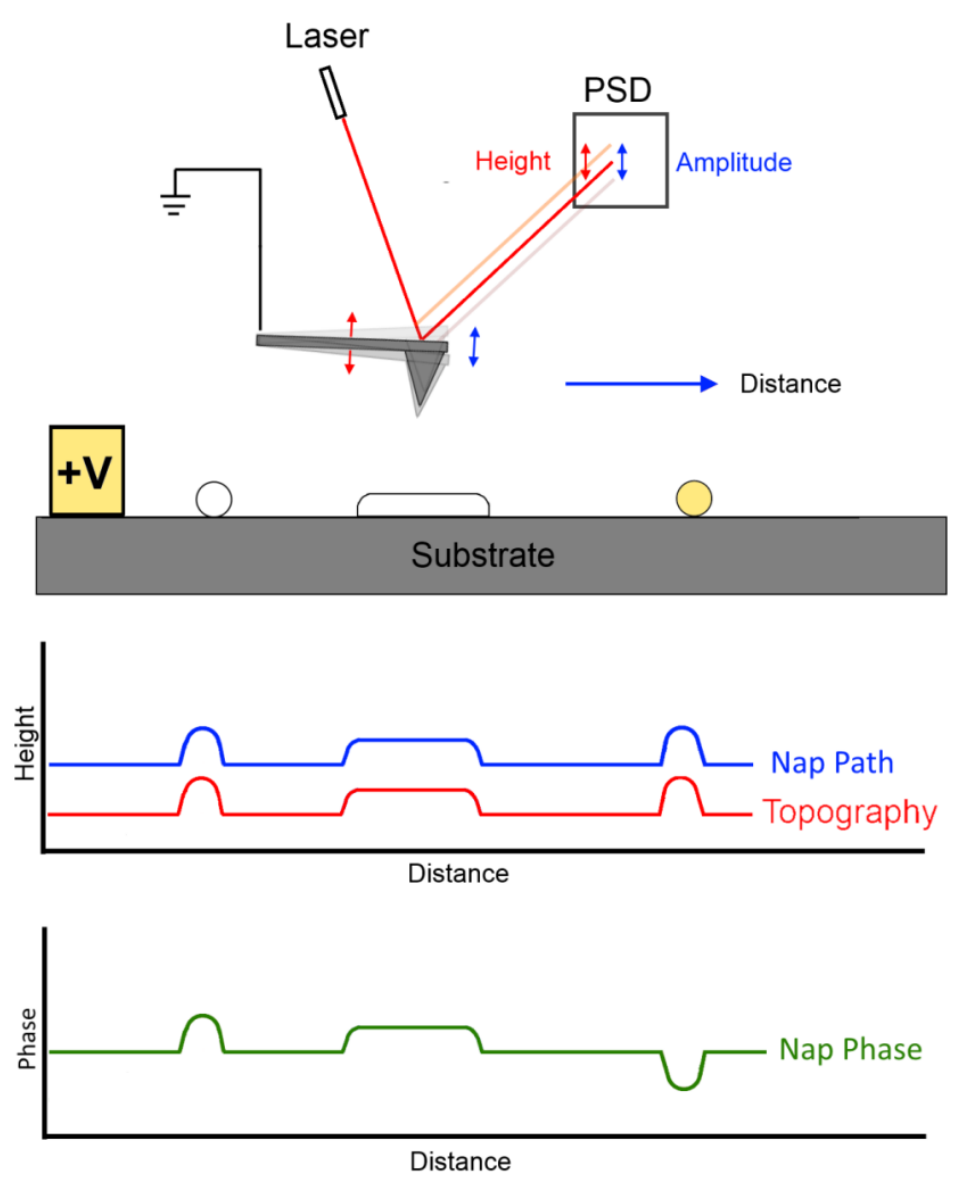

Figure 9: Schematic operation of EFM mode.

\subsubsection{PHASE LOCKED LOOPS WITH EFM}

The AFM uses the PLL to track the oscillation of the tip. The phase measurement of the tip during Nap mode is used to determine the changes in conductivity on the surface. The relationship between the change in conductivity and phase measurement are determined by a phase integrator. The operation of a phase integrator is based on the change in frequency between two signals and is visually demonstrated in Figure 10. The frequency changes generated between the tip and sample offset the phase from the reference oscillation with a time difference of one sinusoid. As the signals continue oscillating, 
the error will compound and linearly change the output phase. For the MFP3D, the slope of the phase is corrected by changing the excitation frequency to match the signal frequency.

\section{Frequency Differences as Phase Integrators}
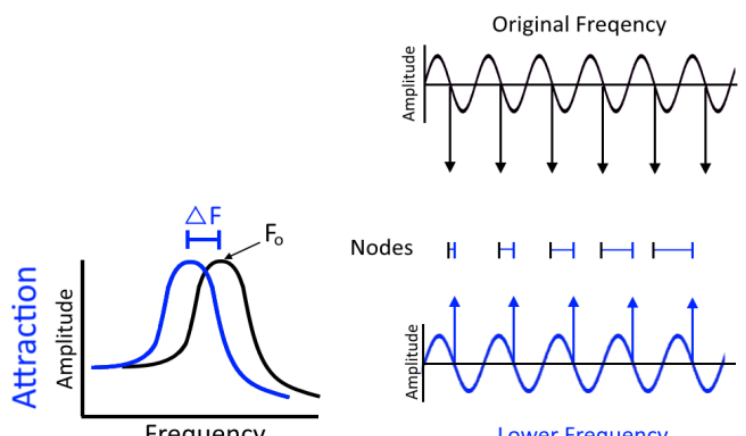

Lower Frequency

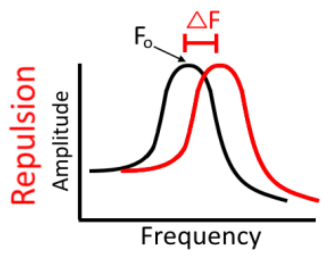

Nodes I H H $\longmapsto \longmapsto \longmapsto$
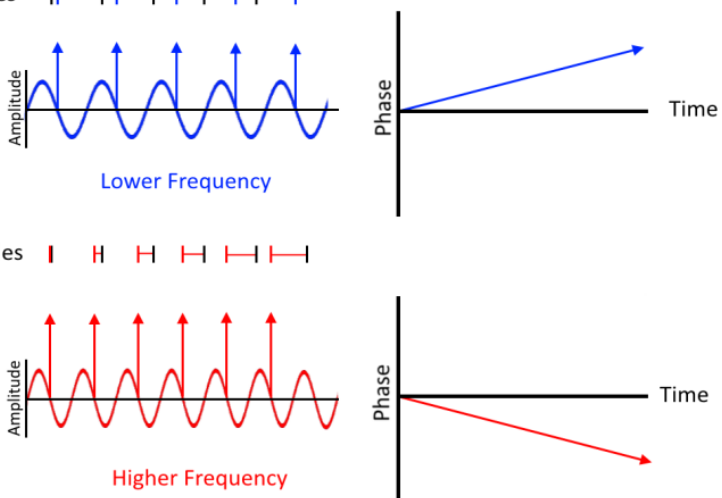

Figure 10: Visual representation of how frequency change from the tip's interactions with the sample creates a linear phase slope.

\subsubsection{AFM PREPARATION}

The AFM used was an Asylum MFP3D AFM with a TS150 noise isolation platform. The vertical resolution is at $0.06 \mathrm{~nm}$, which is sufficient for measuring the topography of thin, surface oxides. The AFM had a conductive mode tip holder termed "ORCA". The ORCA tip holder contains a current amplifier on the tip to allow for measurement of extremely small currents. This current measurement system amplifies the current 100 million times and converts the current to a voltage that the AFM can read. With this ability, the AFM can measure current flow as low as 100pA. 
Preparing the AFM for imaging of the sample begins with installing a tip into the AFM. A low resonance frequency, electrically conductive tip was used for the lithography and imaging. The specific tip used was an Olympus AC240 TM ElectriLeaver. These tips have a $20 \mathrm{~nm}$ Pt conductive coating that consists of $5 \mathrm{~nm}$ Ti adhesion layer to increase the bond strength between the silicon tip and Pt coating. The tips were inserted into a quartz holder with an external biasing wire to apply voltage to the surface of the sample as shown in Figure 11. The tip and holder was inserted into the AFM head to finish assembly. The head contains all the electronics used for operating the AFM. The sample was mounted onto a sample holder with conductive clamps that were connected to the biasing wire shown in Figure 12. The sample was connected to the clamps with colloidal silver to guarantee conductivity.

The operating frequency of the cantilever was then obtained. In software, the machine would perform an oscillation sweep to find the resonant frequency of the cantilever attached to the tip. The user could input the desired operating frequency as a percentage offset from the resonant peak. The decision to use a smaller frequency or larger frequency is based on the desired cantilever behavior. The cantilever will shift its operating frequency as it interacts with the sample. The percent offset from the natural

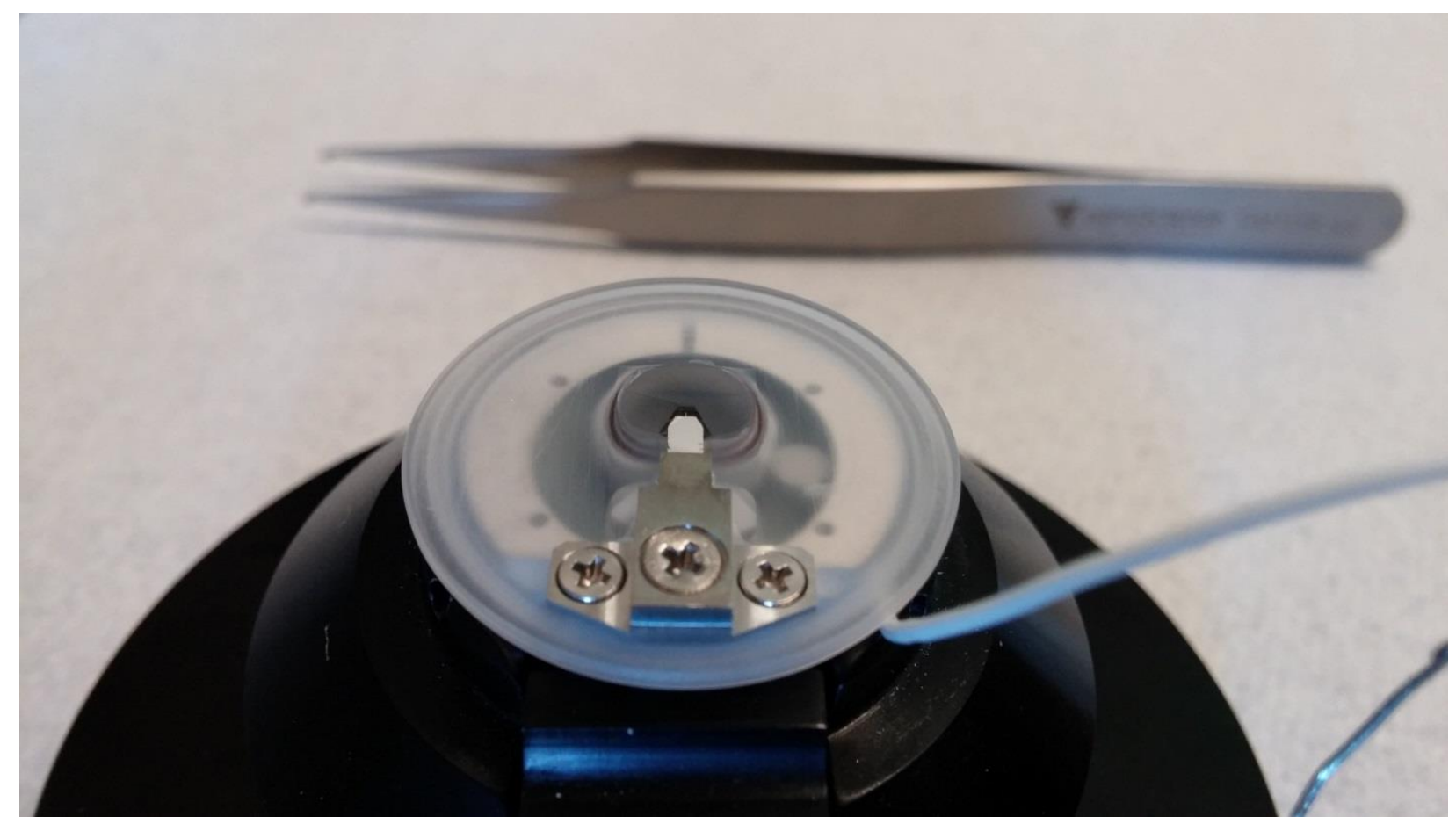

Figure 11: A cantilever inserted into the "ORCA" cantilever holder. 


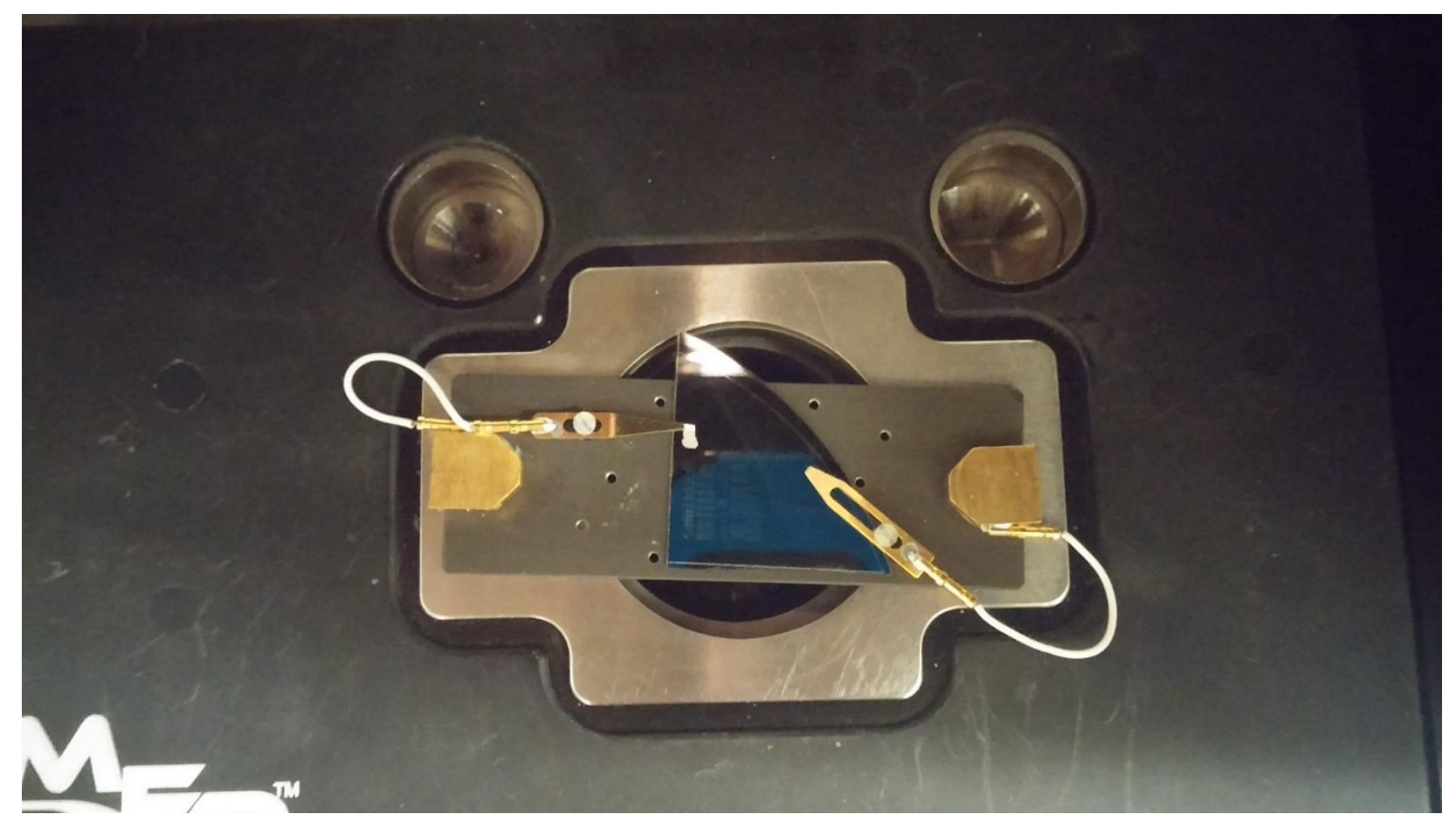

Figure 12: Diced silicon sample with sample holder on the scanning stage.

frequency was chosen for all scans was a frequency $-5 \%$ from the resonant peak. The frequency selected was a lower frequency that was selected to balance signal amplitude and feedback sensitivity for tapping mode AFM. The driving oscillation frequency was then automatically determined by the program.

The software was then configured to allow for scanning to be performed. The parameters that were used to adjust the feedback system were the drive amplitude and the integral gain of the feedback system. The default values set to initially scan was chosen at a slightly increased integral gain, allowing for the feedback system to prevent excessive force on the tip as the user approaches the tip. The tip was then manually lowered using the three screws that the AFM head sat upon. The tip was in scanning distance when the $\mathrm{z}$ feedback system was engaged. A scan would then be initialized by the user. The user slowly increases the drive amplitude until the forward and backwards scan matches. Then, the user can perform optimization of the integral gain to achieve a clear image.

\subsubsection{AFM LITHOGRAPHY PROCEDURE}

The procedure to perform lithography utilized a software module called "Microangelo". The module allowed the user to manipulate the tip while using specified parameters to perform desorption. 
The module accepts both hand drawn and bitmap images to be used to perform specialized potential exposure. The hand drawn method converts the drawn image to a series of vectors in which the tip will follow. The bitmap loading method operates by superimposing a raster scan with potential proportional to the bitmap colors. The applied voltage at each pixel of the scan is set as an interpolated voltage between two colors. For example, if there is a black and white image, the user would input what voltages black and white represent. White represents $0 \mathrm{~V}$ and black represents $1 \mathrm{~V}$, grey pixels of the image would be an interpolated voltage between $0 \mathrm{~V}$ and $1 \mathrm{~V}$, based on how light or dark the grey's color value is. For the hand drawn images, the program allows for the user to draw using a cursor on a scanned image. The drawn lines are converted into editable lithography vectors. Then tip velocity and voltage parameters are set in the program. The user can then start the lithography by confirming the parameters on the program. This method was the most commonly used method due to the speed of operation, ease of use, and individualized lithography that can be drawn, avoiding surface features that influence the lithography.

\subsection{IMAGE PROCESSING}

The topography scans were saved without image modification. However, when looking at such fine features, any tilt can influence the image to hide topography data. When the extrema of the scan are 10 times as large as the largest feature on the silicon, the visibility of the largest feature is 10 times less visible when compared to an image where the scan plane was flat. Post processing methods such as plane fitting and scan line correction can correct for environmental parameters affecting the fidelity of the scan. The program used to perform the corrections was Gwyddion ${ }^{14}$. The plane fitting allows for the flatness of an electromechanically polished silicon wafer to be used as a reference. The roughness of the silicon surface was found to be around $0.15 \mathrm{~nm}_{\mathrm{Rms}}$. The negligible surface roughness makes the passivated silicon a suitable reference that can make a reliable plane for data fitting. This is done by using three averaged points on flat, passivated areas that Gwiddyon uses to fit the data set to the plane made by the points. The size of the sample allows thermal expansion to be able to cause long term drift in the scan. The line scan 
correction is done by matching the median height of all of the scan lines. There is no other modification to the data. From these two steps, the image fidelity was preserved as visibility was enhanced. 


\section{CHAPTER 3: RESULTS AND DISCUSSION}

\subsection{STM RESULTS}

Initial attempts to scan the surface of the passivated silicon under ambient conditions proved to be unsuccessful. A series of voltages ranging from $-2 \mathrm{~V}$ to $2 \mathrm{~V}$ and currents ranging from $0.05 \mathrm{nA}$ to $2 \mathrm{nA}$ were used as set points for STM feedback. Under all set points attempted, the profile did not match sequential scans of the same scan line. In an attempt to determine the reason for the inconsistent profile, voltage sweeps, also known as I-V curves, were performed.

\subsubsection{I-V CURVES}

The I-V curves were used to investigate the inconsistent scan profile. The voltage of the tip was swept from $-2 \mathrm{~V}$ to $0.7 \mathrm{~V}$. The tip was held stationary above the sample after the feedback on the STM adjusted the tip to maintain a signal of $-2 \mathrm{nA}$ at $-2 \mathrm{~V}$. Four scans of various locations within 100 $\mathrm{nm}$ of each other were obtained at different times. Figure 13 shows the I-V curves of the scans superimposed to highlight the variation in electrical behavior between each scan. The scans are labeled according to the time from the first scan. After Time 1, Times 2, 3, and 4 were performed after 8, 13 and 17 minutes respectively. The tip sample interactions were found to be characteristic of a tungsten and $\mathrm{n}$ type silicon interface through the formation of a Schottkey barrier with a height of $0.45 \mathrm{~V}^{15}$. What is evident between the scans is the increase in tunneling barrier between the tip and sample as shown by the reduced tunneling current. Since the operation of the STM cannot perform

analysis of nonconductive materials, the cause for the increase in the tunneling barrier is unknown. A few possible reasons for the change in the tunneling barrier include contaminates on the surface and oxide layers present on either the sample or STM tip. As a result of these findings, AFM was attempted as an alternative method that is not limited by the conductivity of the sample. 


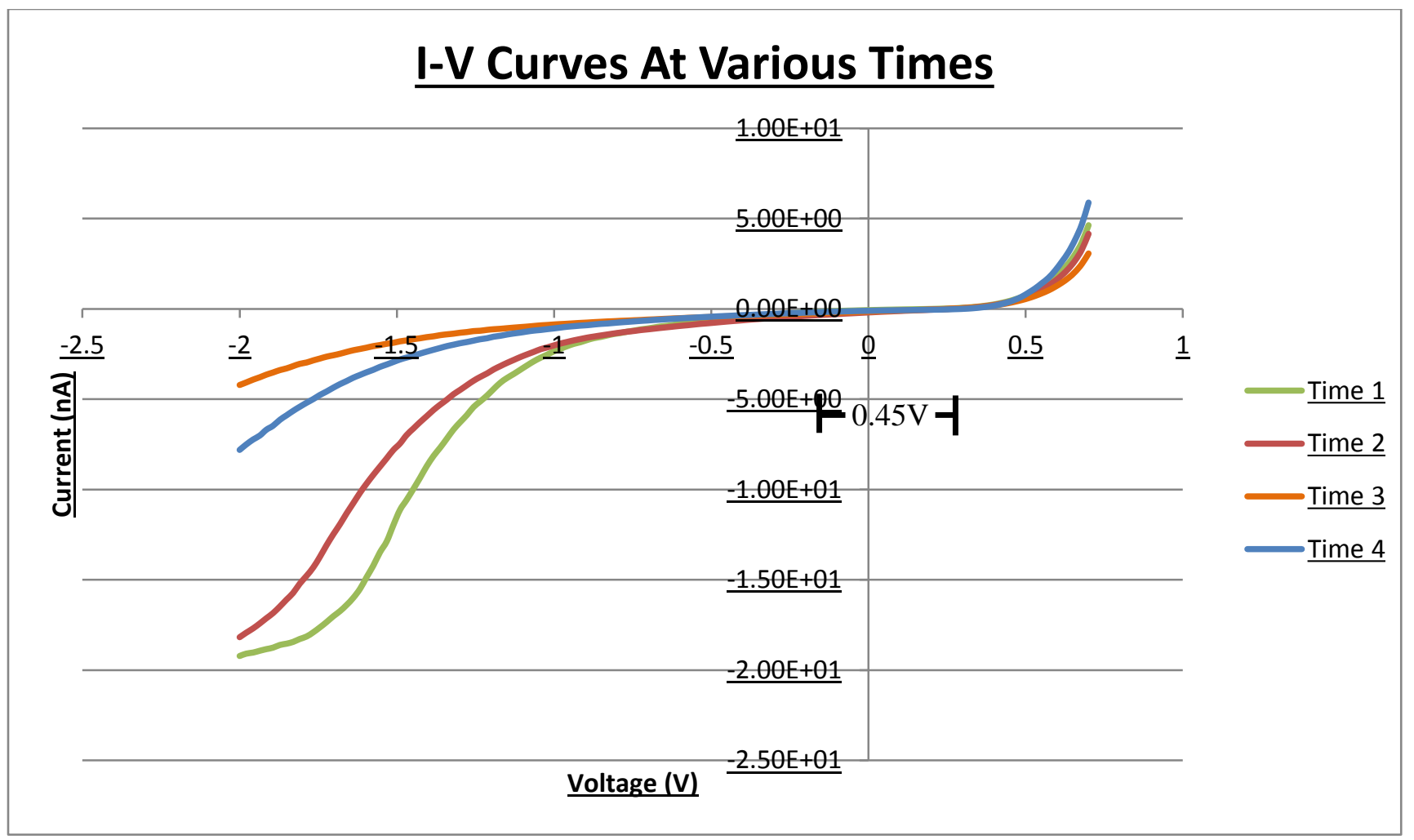

Figure 13: Superimposed I-V curves to compare the gradual increase of electrical resistance. Time 1 represents initial exposure time. Times 2,3,4 were performed at 8, 13, and 17 minutes after the first desorption.

\subsection{AFM I-V CURVES}

I-V spectroscopy using the AFM was used to investigate the electrical interactions between the tip and the sample along with finding the relationship between the voltage applied and the thickness of the produced oxide. Multiple modes of I-V curves were developed to focus on particular aspects of the interactions. The first mode was a simple voltage sweep from a negative voltage to a positive voltage. The resulting current was measured from the ORCA current amplifier. All tips underwent this type of sweep before lithography was performed. The purpose of this method was to determine the band gap of the sample. The second mode of operation was where constant voltages were held for three seconds. The tip was then moved to a new location and the DC voltage was nominally increased. After all the desired voltages were swept through, a tapping mode AFM scan was performed to extract the height profiles from the desorbed areas. Both modes were performed with the tip in contact with the sample surface. 


\subsubsection{AFM I-V SWEEP}

The expected result of a voltage sweep is a smooth curve for both the negative and positive voltages. When the I-V spectroscopy was performed, the result was unexpected. The voltage sweep is shown in Figure 14. The negative applied voltage followed a smooth, predicable curve that can be used to measure the band gap. However, when the potential was switched to be positive, the current appeared normal until a current flow of about 9nA that occurred at about $1 \mathrm{~V}$. After this point, there was only noisy current flow that peaked at $138 \mathrm{nA}$ at $2.52 \mathrm{~V}$. The behavior of the positive voltage sweep made the band gap impossible to measure.

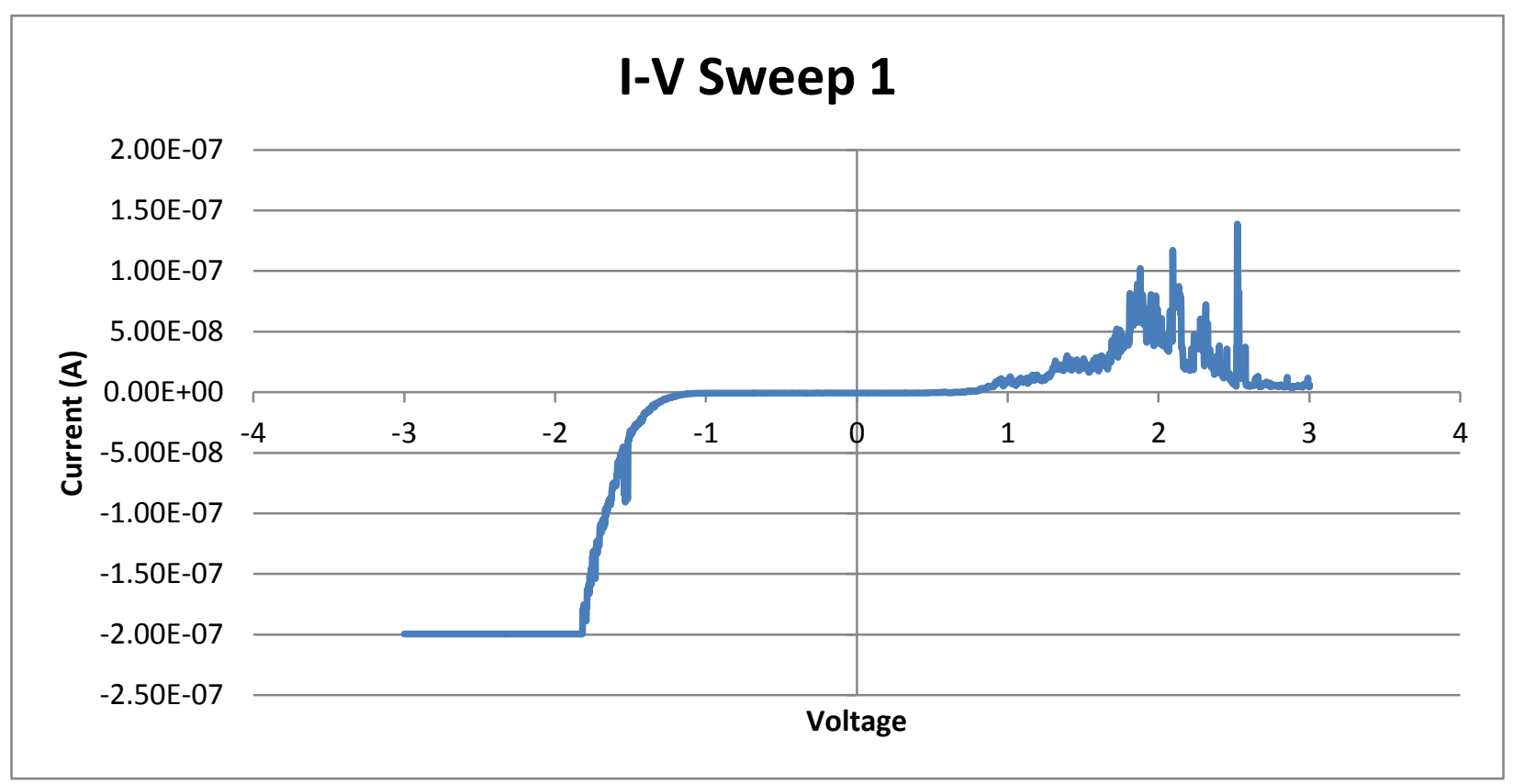

Figure 14: Current vs. Voltage sweep with the voltage applied to the surface, modulated from -3V to $3 \mathrm{~V}$.

Subsequent I-V curves yielded progressively less current when imposed with the same voltage sweep in a new location. The comparison between the first voltage sweep and the next two is shown in Figure 15.The second sweep shows increased resistance between the tip and the sample. On the third spectroscopy, the current flow is almost zero. Additionally, the low current persisted across separate locations on the substrate. This suggests that as with the STM, there was a modification to the tip-sample interface that greatly influenced the electrical properties. After multiple uses of the tip, there was no 
change in the electrical behavior; leading to the conclusion that the tip modification was permanent. Despite the changes to the tip's electrical properties, the tip was still capable of performing desorption.

Desorption events may also be seen in the IV spectroscopy; spikes in current can represent events that occur between the tip and the sample ${ }^{16}$. While the cause of specific events cannot be determined from the I-V sweep, they could represent various tip modification events happening. The modifications could be from the tip acquiring a coating of either an organic contaminate or silicon sputtering to the tip and oxidizing.

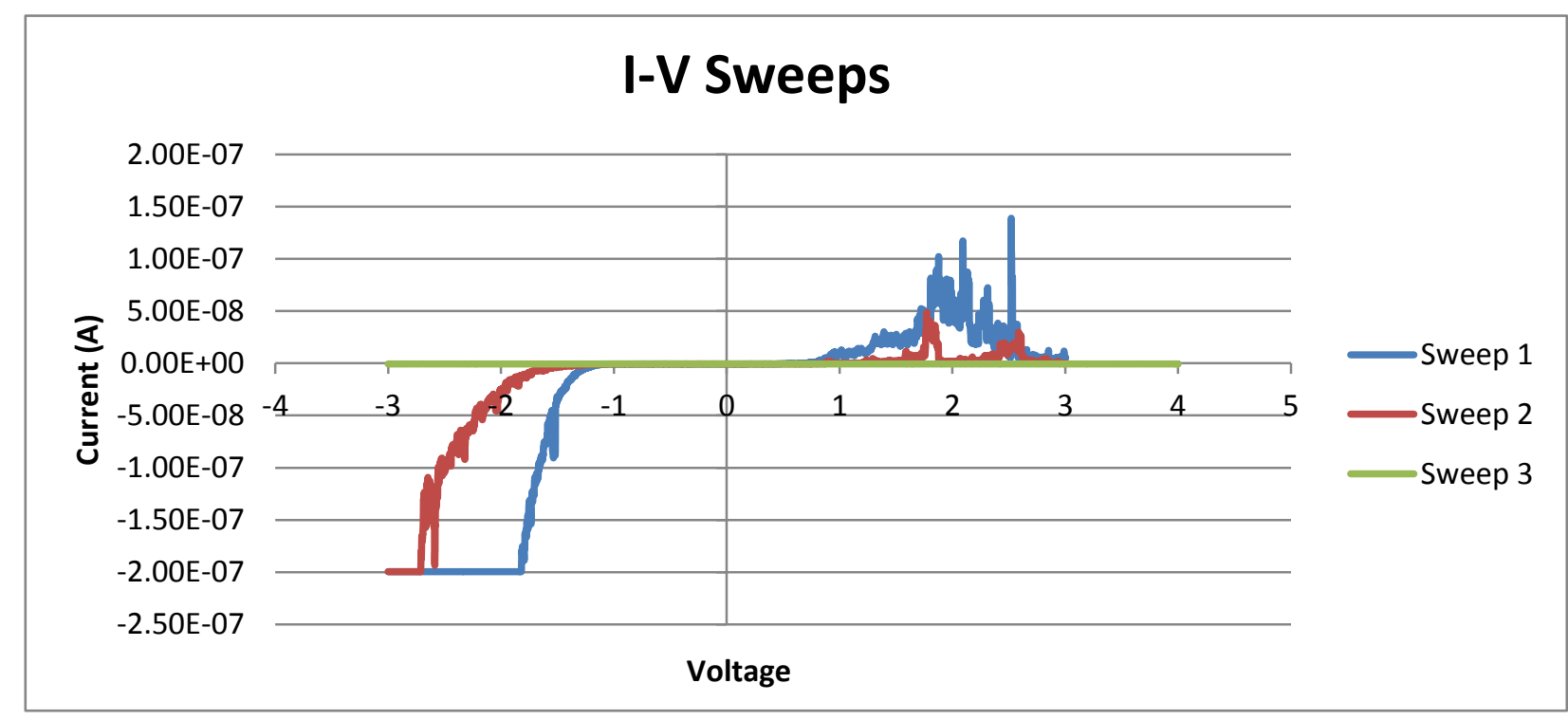

Figure 15: Current vs. Voltage sweeps performed on multiple passivated areas. The sweeps show the process of the tip being modified.

\subsubsection{POINT VOLTAGE SWEEPS}

A fixed DC bias with a superimposed $200 \mathrm{mV}, 2 \mathrm{KHz}$ AC voltage was used to plot the absorbed oxide's thickness compared to the exposure voltage. The results of the chart were compared against literature. The AC wave was intended to be used for further analysis of the desorption current. The stepped DC exposure was performed within a continuous, single area of passivation. Two sets of sweeps were performed on separate samples. The first sweep was a broad sweep that covered both positive and negative voltage. The second sweep measured a smaller voltage range with a finer voltage step. All spectroscopies were exposed for three seconds to ensure sufficient exposure to the surface along with the 
same electron dose. The measured current when exposing each point was less than 1nA. Each DC voltage was applied with a separation of 500nm in a grid system. The points of the grid were chosen to avoid existing surface features. Profiles of each protrusion were extracted and analyzed investigate the desorption height at each voltage.

\subsubsection{I-V SPECTROSCOPY RESULTS}

The first set of sweeps performed covered a voltage range of $-7 \mathrm{~V}$ to $7 \mathrm{~V}$. The sweep is defined as coarse because voltage was incremented in steps of 0.5 volts. A topographic scan of positive and negative exposure results along with an EFM scan of the negative exposure are shown in Figure 16. The negative voltage sweep resulted in no distinguishable desorption. However, an EFM scan of the same area shows the points were charged. The exposure voltage that yielded a measurable oxide was for voltages above 4.5V. While the negative voltage never desorbed from the surface, residual charging was recorded with an

a)

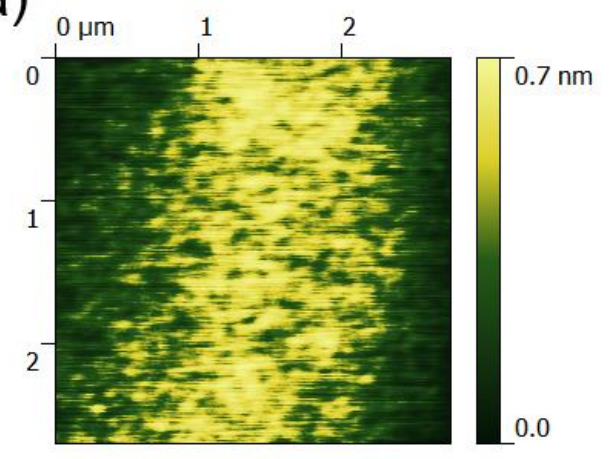

b)

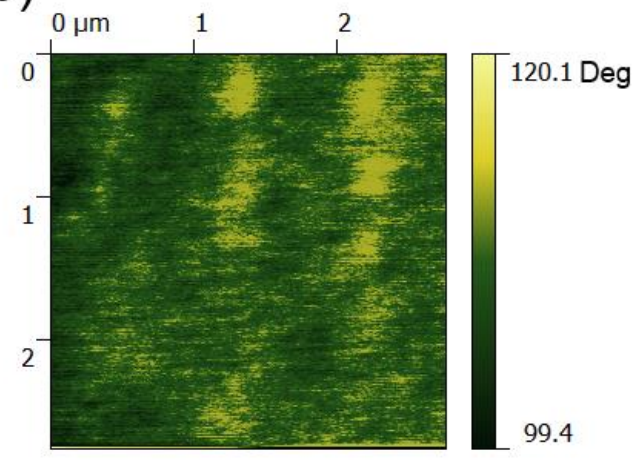

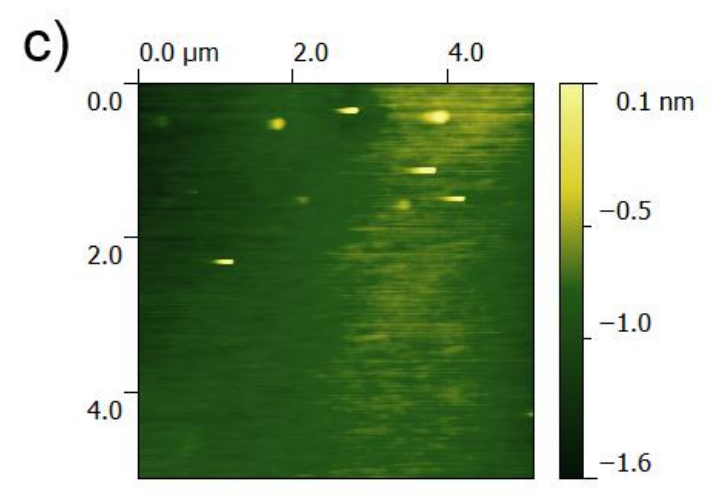

Figure 16: Scans after performing coarse voltage point spectroscopy. The topography after applying negative voltage a) showed no presence of oxide. b) An EFM scan shows there was residual charge. c) The topography of the positive voltage desorption 
EFM scan. The charging on the surface was found to be larger with larger negative voltage. This suggests that the sample might have been partially desorbed when performing the spectroscopies.

The second set of sweeps was categorized as fine since it was performed with a voltage range of $3 \mathrm{~V}$ to $7 \mathrm{~V}$ in increments of $0.2 \mathrm{~V}$. The resulting tapping mode image of the spectroscopy is shown in Figure 17. At all potentials tested, the AFM was able to detect oxide layers present.

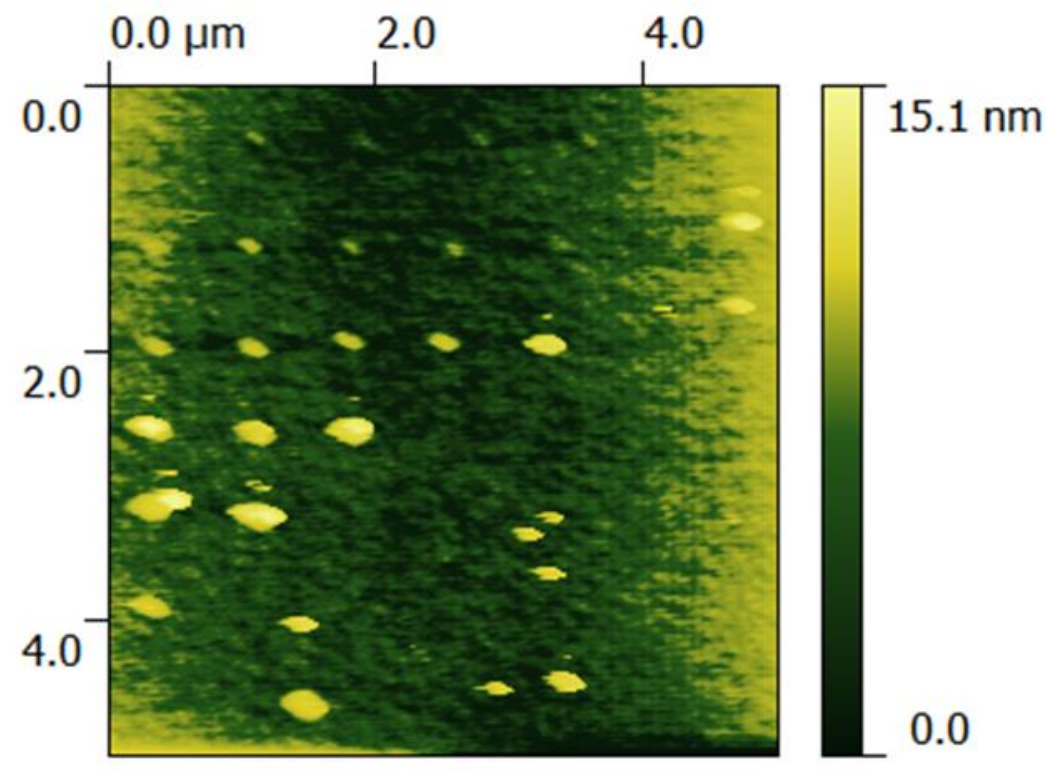

Figure 17: Tapping mode scan after performing coarse voltage point spectroscopy.

The measured oxide thickness compared to the exposure voltage for both the scans is shown in

Figure 18. There were distinct differences between the $0.5 \mathrm{~V}$ incremented sweep and the $0.2 \mathrm{~V}$

incremented sweep. From the coarse sweep, the current values were significantly less thick than expected. For example, the $5 \mathrm{~V}$ desorption thickness were $0.2 \mathrm{~nm}$ and $0.5 \mathrm{~nm}$ when in literature, the desorption thickness was found to be closer to $1 \mathrm{~nm}^{8}$. The cause for the reduced thickness is possibly due to a partially desorbed surface. For the fine sweep voltages above $5.6 \mathrm{~V}$, the height of the oxide spikes to more than double the thickness of the established trend. The thickness of the oxide ranging from 8 to $12 \mathrm{~nm}$ was thicker than what is presented in literature for AFM and is closer to what was expected for an optical desorption system ${ }^{10}$. The varying behavior of the oxide thickness suggested that the tip may have been 
switching from using primarily tunneling current to using field emission. From the data presented, it was shown that there were influences on the oxide thickness achieved that showed no distinct pattern.

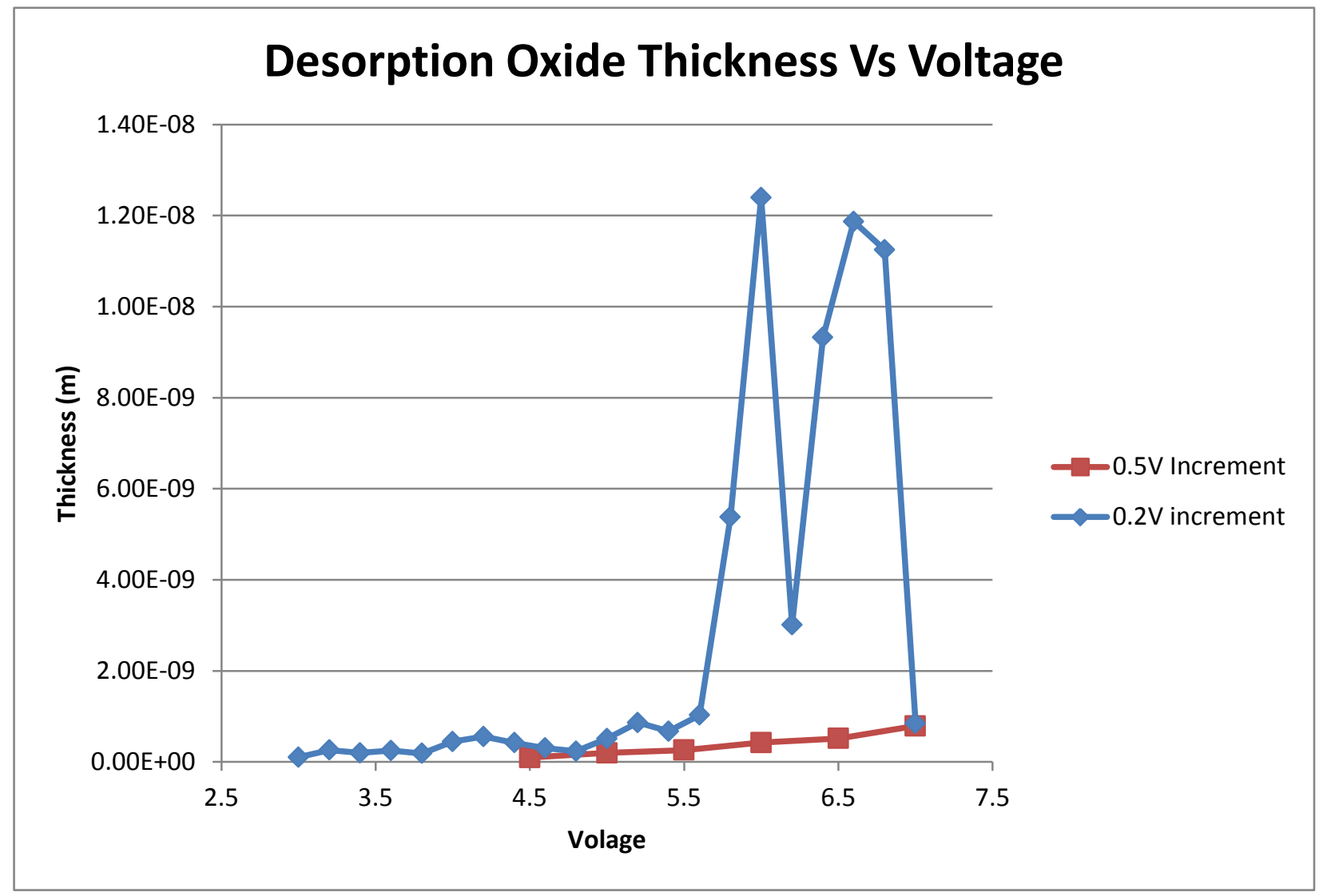

Figure 18: Extracted Height profiles from both the rough and fine voltage sweeps.

\subsection{DESORPTION MEASUREMENTS}

A test image of the CP logo was drawn using the Microangelo program using an excitation voltage of $5 \mathrm{~V}$. After the contact mode lithography was performed, the area was scanned using the AFM in tapping mode. The resulting scan is shown in Figure 19a. The target area of the passivated silicon successfully desorbed the hydrogen and absorbed oxide in the locations determined by the Microangelo program. A line profile of data was extracted from the image of the oxide writing as shown in Figure 19b. The extracted line profile yielded the height and width of the formed protrusion. Based on the line profile extracted from the image, the height and width was found to be $0.912 \mathrm{~nm}$ and $79.02 \mathrm{~nm}$ respectively 
(Figure 19b). The height of the lines corresponded to previously measured oxide heights of about $1 \mathrm{~nm}$ for a desorption voltage of $5 \mathrm{~V}^{8}$. It was concluded that using the Microangelo program with the tip in contact mode could successfully grow a patterned oxide mask.

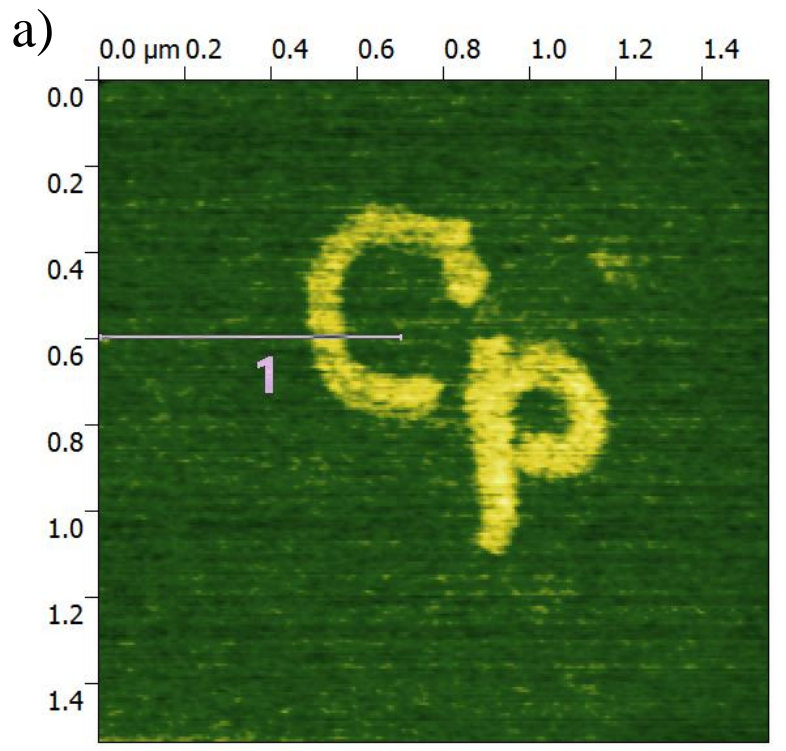

b)

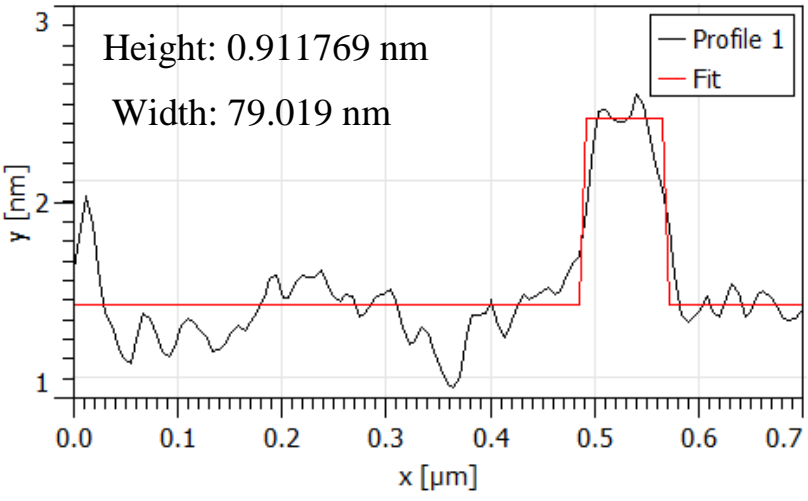

Figure 19: Cal Poly logo drawn though desorption. a) The topography of the scan is shown along with the location for the line profile extraction. b) The corresponding step height measurement.

\subsubsection{FORCES FROM TIP CHARGES}

The presence of charge attraction between tip and sample was noticed when scanning silicon.

When the tip tried to image the surface in the attractive regime (Phase<90), the tip would snap to a low amplitude readout and produce a poor image. The scan lines would not match when rastered across the same area, suggesting the acquired data is not accurate. However, when the cantilever was brought to be in the repulsive or tapping mode, the tracking properly recreated the surface. This behavior suggests that an attractive layer formed between the tip and the sample. Under ambient conditions, it is believed that a moisture layer forms across the surface of the silicon. The surface tension of water on the surface would force the tip to be in contact with the surface of the passivation layer when attempting noncontact mode AFM. The snap down while imaging in attractive mode suggests that there is a presence of a moisture layer in the system being evaluated. 
The tapping mode topography scans gave an initial assessment to the desorption system being evaluated. Three factors were revealed from AC mode imaging: the presence of a moisture layer, surface growth after performing lithography procedure, and the tip modification during lithography. Additional investigation was performed to further characterize the lithography capability.

\subsection{ELECTRONIC FORCE MICROSCOPY VERIFICATION}

The electronic force microscopy was performed on the desorption layer to verify the properties of the oxide. EFM allows for the conductance of materials to be determined as a part of an image. The primary function of EFM allows for relative conductivities to be distinguished on the surface of the sample. This method was used as a secondary detection method that confirmed desorption regions were comprised of an oxide and not contaminates.

\subsubsection{EFM ASSUMPTIONS}

The proposed method of operation describes how the charges between the tip and the sample interact. Conductive and insulating particles on the surface of the sample affect the phase of an oscillating tip through charge interactions. The method uses Coulomb's Law: charges with opposite sign will attract and charges with like sign will repel. The conductive tip was connected to ground and assumed to always have a negative surface charge that interacts with the sample surface. The charge mobility in the materials is assumed to be proportional to the conductivity of the material. Charges in insulators are not mobile and can only orient themselves to create a dipole. Charges in a semiconductor are semi-mobile and will have limited movement. Charges in conductors are assumed to be extremely mobile.

\subsubsection{POSITIVE VOLTAGE EFM}

Utilizing the assumptions about charge and charge mobility, the operation of EFM with a positive surface voltage is shown in Figure 20. The positive applied voltage to the substrate will increase the negative charge density at the contact area. The gathering of negative charges creates positive charge area across the rest of the sample. When the tip is interacting with the semiconductor, a baseline tip sample 
force is established. This force has an attractive force between the tip and sample, due to the presence of opposite charges between the surface and the tip. When there is an insulator between the tip and substrate, the charges cannot move and are limited to reorienting themselves. There is an increased concentration of negative charges in the material when compared to the surrounding area, causing an increased repulsion force. The increased force between the tip and the insulator causes a positive phase shift measured by the PLL. When there is a conductor between the tip and the substrate, the charges are extremely mobile. As a result, a small layer of positive charges are formed on the surface while the negative charges are repelled from the surface. The small positive layer of charges will move to the area influenced by the tip and track along with the tip due to the high mobility of the electrons. The large local presence of positive charges

\section{Positive Surface Voltage EFM}

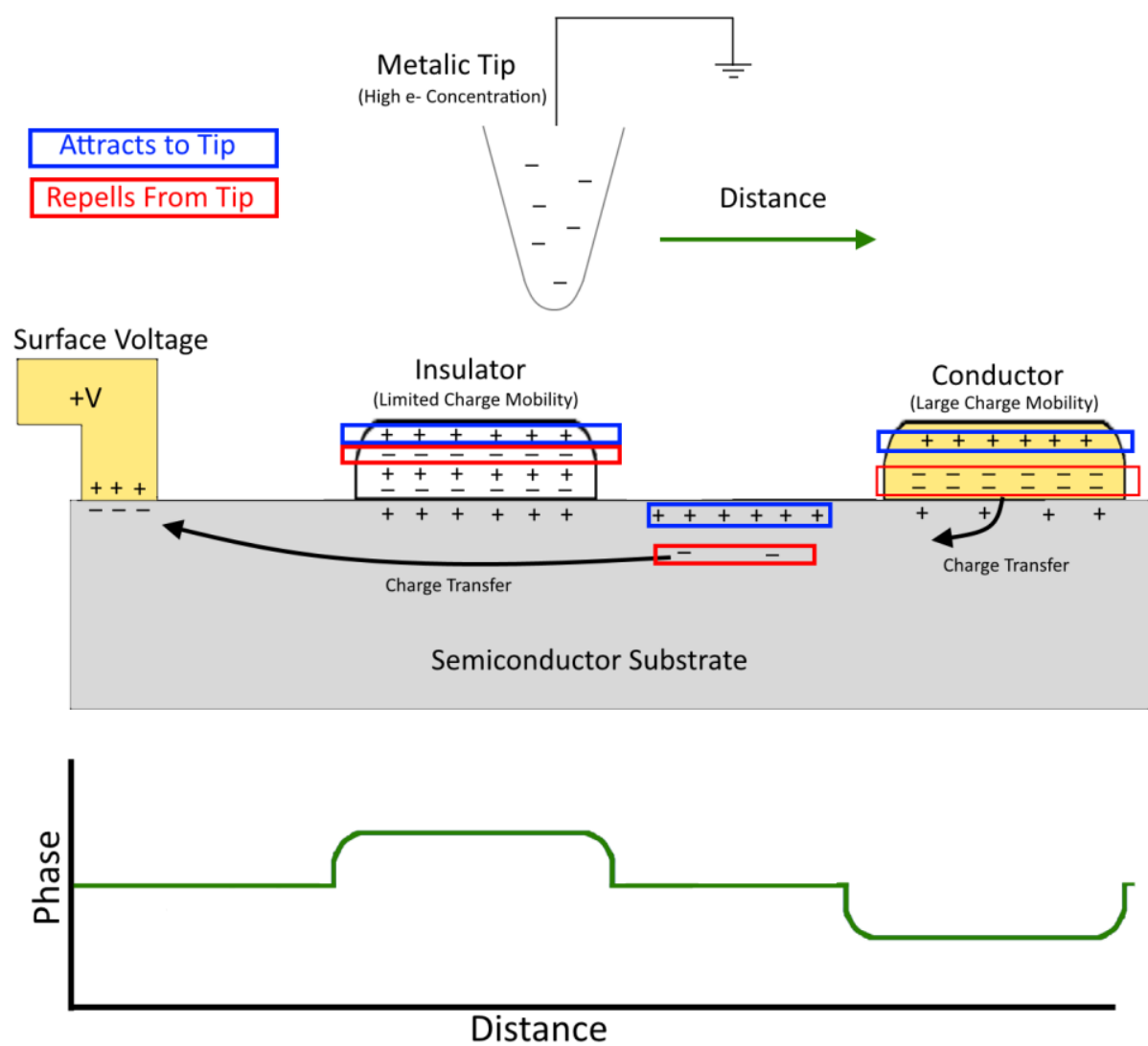

Figure 20: Charge interaction between the tip and sample when performing EFM with a positive surface bias. 
creates a larger attraction force on the tip. The forces are picked up by the PLL and result in a negative phase shift.

\subsubsection{NEGATIVE VOLTAGE EFM}

The operation of EFM with a Negative surface voltage similar to the positive voltage EFM and is shown in Figure 21.The difference between the operation is the charge distribution along the surface. The charges are reversed, but the tip remains as a high concentration of electrons. This time, the insulator orients its self so that the negative charge is on the surface. The conductor will have a presence high concentration of electrons on the surface. The tip interactions between conductor and tip invert, along with the insulator and tip interactions. As a result of the charges switching, the images created with the

\section{Negative Surface Voltage EFM}

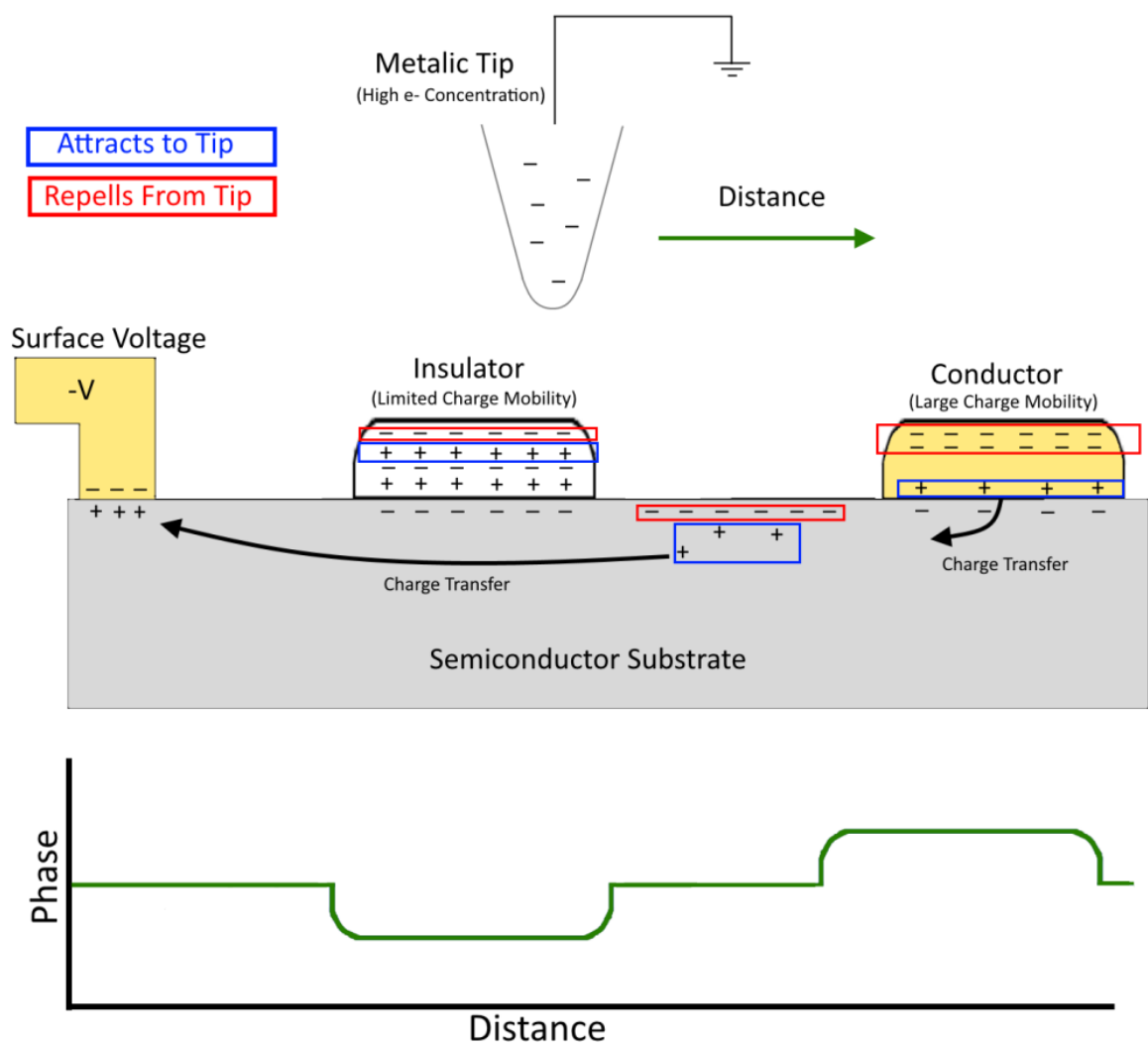

Figure 21: Charge interactions between the tip and sample when performing EFM with a negative surface bias. 
negative bias created an image in which the insulator shifts the phase down while a semiconductor would shift the phase up.

\subsubsection{EFM RESULTS}

The nap phase images that were analyzed were performed at a transition between passivated silicon and on a grown oxide layer. A series of applied voltages were performed to confirm the desorbed section as an insulator. A series of voltages of $-2 \mathrm{~V}, 0 \mathrm{~V}$ and $1 \mathrm{~V}$ were applied to analyze the response to test the sample. The zero voltage was used as a control to verify that the Van Der Waals forces were not influencing the scan. The results of the scans are shown in Figure 22.The relative heights of the areas between the scans were compared. The control scan showed that the tip was sufficiently separated from the surface to prevent influence from the height change of the step. The negative applied surface voltage showed a positive shift in the phase on the desorbed surface and a negative phase shift on the substrate, which suggests the desorbed surface was more of an insulator. The positive voltage applied resulted in a negative phase shift on the desorbed surface and a positive phase shift on the substrate. The phase shift directions of the positive applied voltage also suggest that the desorbed surface was an insulator. 


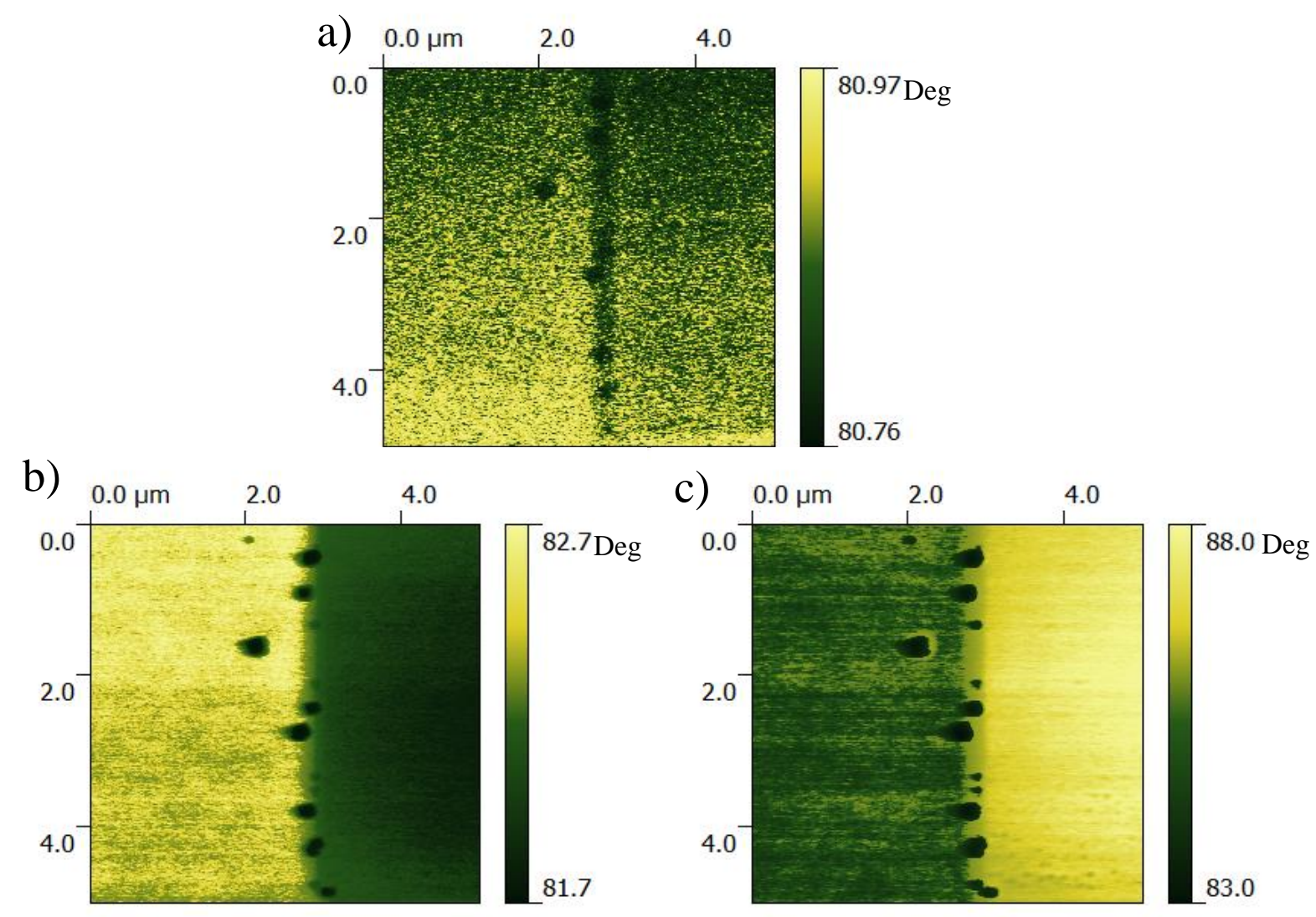

Figure 22: EFM images on the edge of a desorbed square. The left side of the step is the semiconductor while the right is a part of the desorbed square. The EFM images correspond to a surface voltage of a) $0 \mathrm{~V}, \mathrm{~b}$ ) $-2 \mathrm{~V}$, and c) $1 \mathrm{~V}$. 


\section{CHAPTER 4: FUTURE WORK}

The scope of this paper was to investigate the growth of an oxide layer. The next step to this project is to investigate etching the silicon nanowires. The methods that would be investigated would be to use both wet and dry etching methods to etch the mask with. Once a characterized etch procedure is obtained, transistors can be developed to find the optimal size for the nanowire to produce high speed and efficient transistors. 


\section{CHAPTER 5: CONCLUSIONS}

Overall, a successful process has been developed for manufacturing silicon nanowires. The STM analysis proved to give inconclusive results for the configuration used. The results showed that an oxide layer was growing too fast to image. The AFM was shown to have limited success. While desorption was successfully performed, the trace width was found to be $80 \mathrm{~nm}$ which is larger than the nanowire transistor presented in literature. There are mentions in literature where the tip's electronic profile can be modified by adjusting the height of the tip to change the distributions ${ }^{5}$. The thicknesses obtained from the point spectroscopy seemed to follow an interesting trend where the oxide thickness would vary greatly with voltage. The desorption capability was not affected by heavy modifications to the tip that inhibited current flow. The developed procedures and analysis enables a designer to devise a more precise solution to the construction of nanowire transistors with scanning probe technology. 


\section{REFERENCES}

1. Bohr, M. \& Mistry, K. Intel's Revolutionary $22 \mathrm{~nm}$ Transistor Technology. at <http://download.intel.com/newsroom/kits/22nm/pdfs/22nm-details_presentation.pdf>

2. McKendry, R. et al. Creating Nanoscale Patterns of Dendrimers on Silicon Surfaces with Dip-Pen Nanolithography. Nano Lett. 2, 713-716 (2002).

3. Wang, W. M., Lemieux, M. C., Selvarasah, S., Dokmeci, M. R. \& Bao, Z. Dip-Pen Nanolithography of Electrical Nanotubes. 3, 3543-3551 (2009).

4. Martínez, R. V, Martínez, J. \& Garcia, R. Silicon nanowire circuits fabricated by AFM oxidation nanolithography. Nanotechnology 21, 245301 (2010).

5. Snow, E. S. \& Campbell, P. M. Fabrication of Si nanostructures with an atomic force microscope. Appl. Phys. Lett. 64, 1932 (1994).

6. Campbell, S. Fabrication Engineering. (Oxford University Press, 2008).

7. Miura, T., Niwano, M., Shoji, D. \& Miyamoto, N. Kinetics of oxidation on hydrogenterminated Si(100) and (111) surfaces stored in air. J. Appl. Phys. 79, 4373 (1996).

8. Snow, E. S., Jernigan, G. G. \& Campbell, P. M. The kinetics and mechanism of scanned probe oxidation of Si. Appl. Phys. Lett. 76, 1782 (2000).

9. Stiévenard, D. \& Legrand, B. Silicon surface nano-oxidation using scanning probe microscopy. Prog. Surf. Sci. 81, 112-140 (2006).

10. Oxidation of hydrogen-passivated silicon surfaces by scanning near-field optical lithography using uncoated and aluminum-coated fiber probes.

11. Shen, T.-C. \& Avouris, P. Electron stimulated desorption induced by the scanning tunneling microscope. Surf. Sci. 390, 35-44 (1997).

12. Stokbro, K. et al. STM-Induced Hydrogen Desorption via a Hole Resonance. 3-6 (1998).

13. Harris, R. Modern Physics. (Pearson / Addison Westly, 2008).

14. Gwyddion - Free SPM (AFM, SNOM/NSOM, STM, MFM, ...) data analysis software. at $<$ http://gwyddion.net/>

15. Metal-Semiconductor Ohmic \& Schottky Contacts / Barrier Height and Depletion Width Calculators. at <http://www.cleanroom.byu.edu/ohmic-schottky.phtml> 
16. Soukiassian, L., Mayne, A., Carbone, M. \& Dujardin, G. Atomic-scale desorption of H atoms from the $\mathrm{Si}(100)-2 \times 1: \mathrm{H}$ surface: Inelastic electron interactions. Phys. Rev. B 68, 035303 (2003). 